\title{
Influence of Heterogeneous Contact Stiffness and Heterogeneous Friction Coefficient on Frictional Squeal
}

\author{
Lijun Zhang $\left(\mathbb{D},{ }^{1,2}\right.$ Wenbo Li $(\mathbb{D})^{1,2}$ and Dejian Meng $\mathbb{D}^{1,2}$ \\ ${ }^{1}$ College of Automotive Studies, Tongji University, Shanghai 201804, China \\ ${ }^{2}$ Collaborative Innovation Center for Intelligent New Energy Vehicle, Tongji University, Shanghai 201804, China \\ Correspondence should be addressed to Dejian Meng; mdj0218@163.com
}

Received 10 July 2017; Revised 4 January 2018; Accepted 7 February 2018; Published 22 March 2018

Academic Editor: Tai Thai

Copyright (c) 2018 Lijun Zhang et al. This is an open access article distributed under the Creative Commons Attribution License, which permits unrestricted use, distribution, and reproduction in any medium, provided the original work is properly cited.

\begin{abstract}
Contact stiffness and friction coefficient are excitation sources and key influencing factors to frictional squeal with obvious inhomogeneous characteristic that is always neglected. In this paper, a multipoint contact flexible pin-on-disc system is established considering tangential stiffness. Then influence of contact stiffness and friction coefficient with heterogeneous distribution on frictional squeal is studied using the complex modal analysis. The research shows that contact stiffness and friction coefficient heterogeneities influence the likelihood of occurrence of the squeal, the frequency of the squeal, and the real part of the complex eigenvalue of the system. And when the contact stiffness and friction coefficient are close to the boundary of the region of modecoupling instability, flexible pin-on-disc system with homogeneous contact stiffness and friction coefficient cannot predict whether frictional squeal occurs or not. Besides, uncertain distribution of contact stiffness and friction coefficient can induce the uncertainty of brake squeal.
\end{abstract}

\section{Introduction}

Frictional squeal has high frequency and high intensity and it exists widely in various mechanical systems such as brake system, clutch, and train wheel/rail $[1,2]$. It may give rise to enormous economic losses, and it has long been a major concern for the mechanical engineering [1-4]. Till now, it shows that heterogeneous surface topography and material property play a key role in the local contact stiffness and friction coefficient and further greatly influence the frictional squeal [5-8]. So it is of great importance to study the influence of contact stiffness and friction coefficient with heterogeneous distribution in contact surface on frictional squeal.

Many numerical approaches have been presented to explore the squeal phenomenon with deterministic parameters. The influence of the friction coefficient and contact stiffness was always investigated based on analytical models [9, $10]$ and finite element models $[11,12]$, and it has been accepted that high values for friction coefficient lead to high levels of dynamic instability $[13,14]$. However, researches focus more on the influence of mean value of friction coefficient and integral contact stiffness, and the heterogeneities of friction coefficient and contact stiffness are neglected. Some studies investigated the influence of heterogeneous contact stiffness on frictional squeal but heterogeneous friction coefficient was neglected [15]. This hinders further understanding of the mechanism of frictional squeal and reduced the prediction accuracy of frictional squeal frequency.

Recently, some researches aimed at applying uncertainty analyses to frictional squeal. Renault et al. [16] investigated brake squeal uncertainty based on finite element model using interval parameter method, and the parameters of working load, damping property effects, material property effects, and pad surface topography effects were treated as uncertain parameters; Sarrouy et al. [17] proposed polynomial chaos expansions to study brake squeal uncertainty and the stability of a finite element model of a brake was investigated when its friction coefficient or the contact stiffness was treated as random parameter; Hui Lü proposed a hybrid uncertain model with random and interval parameters to deal with the uncertainties existing in a disk brake system, and Young's modulus of the pad was treated as random parameter [18, 19]; Culla and Massi [20] investigated the statistical 


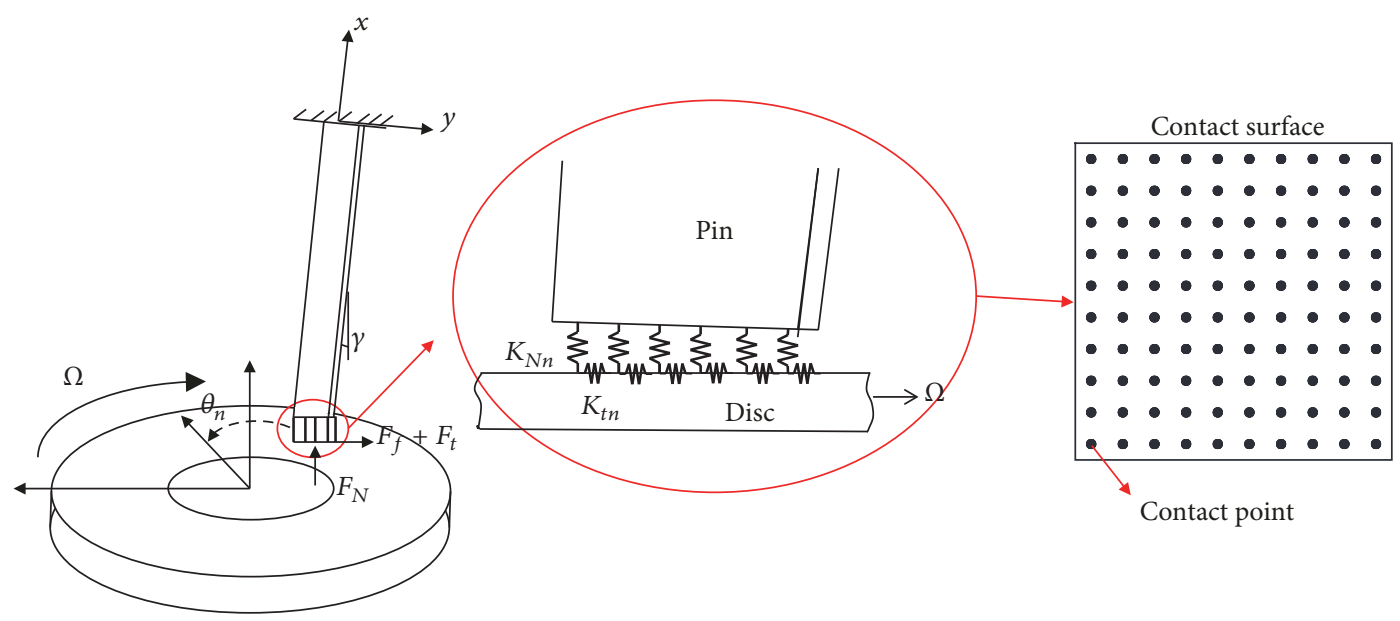

(a) Flexible pin-on-disc model

(b) Schematic of contact area

(c) Schematic of contact points

FIGURE 1: Multipoint contact pin-on-disc model and schematic of contact points.

characteristics of real part of the complex eigenvalue of the system under the assumption that its friction coefficient was random parameter; then a simplified model was established to save computation time; Nobari et al. [21] proposed an efficient method of uncertainty propagation via surrogate modeling and then the statistical characteristics of frequency and real part of the complex eigenvalue of an unstable mode were studied; Sarrouy et al. [22] proposed two methods based on the polynomial chaos to carry out the stochastic study of a brake squeal and investigated the statistical characteristics of frequency and real part of the complex eigenvalue of unstable mode when friction coefficient and contact stiffness were treated as uncertain parameters, respectively. Former studies show that the uncertainties of friction contact parameters are important causes of frictional squeal uncertainty. While only the influence of mean value of friction coefficient and integral contact stiffness is analyzed, the uncertainties of the friction contact parameters distribution are often neglected. In fact, with the same mean value of contact stiffness and friction coefficient, uncertainties of the friction contact parameters distribution can also induce frictional squeal uncertainty.

Consequently, the objective of this paper is to present an analytical model including contact stiffness and friction coefficient heterogeneities in the contact surface. This numerical model is a multipoint contact flexible pin-on-disc model with tangential stiffness in consideration. Focus is put on the influence of the contact stiffness and friction coefficient with heterogeneous distribution on the likelihood of squeal occurrence via mode lock-in. Besides, the probability density curves of the unstable frequencies and real parts of the complex eigenvalues of the system are analyzed with contact stiffness and friction coefficient obeying normal distribution, respectively.

\section{Multipoint Contact Flexible Pin-on-Disc Model and Complex Modal Analysis}

2.1. Modeling. The pin-on-disc system used in the present study consists of 2 components, (i) a flexible pin and (ii) a flexible disc, as shown in Figure 1(a). The disc is assumed to be clamped at the inner bore and free at the outer rim. $r_{\text {in }}$ is the radius of the inner bore and $r_{\text {out }}$ is the radius of the outer rim. The pin has a uniform square cross-sectional area whose side length is $a_{p}$ and it is assumed to be fully clamped at one end and in contact with the disc at the other end. One end of the pin is in contact with the disc, which rotates at the constant speed of $\Omega$, with multiple contact points. The pin inclines with an angle of $\gamma$ along the direction of rotation of the disc.

In developing the analytical modeling, the following assumptions are introduced: (1) The pin can vibrate in two principal directions: the axial direction, $x$, and the transverse direction, $y$. (2) The disc can vibrate only in the out-of-plane direction, $w$. (3) Since disc brake squeal tends to appear at low speeds, centripetal and gyroscopic effects may be omitted. (4) The effect of friction follower force is neglected on the basis that contact stiffness term is much larger than preload term in the stiffness matrix. (5) All contact points are in contact and the shape of the contact surface is not changed in the process of the disc rotation.

There are three coordinate systems in the model, which are the absolute coordinate systems $(\widetilde{r}, \widetilde{\theta})$, the coordinate systems $(r, \theta)$ fixed on the disc, and the coordinate systems $(x, y)$ fixed on the pin, respectively.

2.1.1. Contact Forces. To study the influence of the heterogeneous contact stiffness and friction coefficient on frictional squeal, multiple contact points are introduced to the model. The free end of the pin is assumed to be in constant contact with the disc with 100 contact points. Each contact point is set as follows: divide the contact surface into 100 small squares, and then each contact point is set on the geometric center of each square as shown in Figure 1(c). The geometric center of the contact surface is $\left(r_{0}, 0\right)$. There are three kinds of forces on each contact point, which are normal contact force $F_{N n}$, tangential contact force $F_{t n}$, and friction force $F_{f n}$, where $n$ represents the number of contact points: $n=1,2,3, \ldots, n_{1}$. To simplify the study, normal contact and tangential contact are assumed as linear springs as shown in Figure 1(b). And 
the normal contact stiffness on contact point $n$ is $K_{N n}$, the tangential contact stiffness is $K_{t n}$, and the friction coefficient is $\mu_{n}$. The normal contact force is assumed as a function of relative transverse displacement between pin and disc:

$$
\begin{aligned}
& F_{N n}=K_{N n}\left(-u^{a}\left(l_{P}, t\right) \cos \gamma+u^{t}\left(l_{P}, t\right) \sin \gamma\right. \\
& \left.\quad+w\left(r_{n}, \alpha_{n}(t), t\right)\right)+F_{L n},
\end{aligned}
$$

where $u^{a}\left(l_{P}, t\right)$ is the displacement in the axial direction on each contact point, $u^{t}\left(l_{P}, t\right)$ is the displacement in the transverse direction on each contact point, and $w\left(r_{n}, \alpha_{n}(t), t\right)$ is the displacement in the out-of-plane direction on each contact point.

Tangential contact force on each contact point is

$$
F_{t n}=K_{t n}\left(u^{a}\left(l_{P}, t\right) \sin \gamma+u^{t}\left(l_{P}, t\right) \cos \gamma\right) .
$$

The friction force on each contact point is

$$
F_{f n}=\mu_{n} F_{N n} \text {. }
$$

Assuming that the ratio of the tangential stiffness to the normal stiffness between two bodies is a constant value $\xi$ [5] on each contact point, the tangential stiffness can be written as

$$
K_{t n}=\xi K_{N n}
$$

2.1.2. Modeling of the Flexible Disc. Assuming that, at the beginning, the coordinate of the free end of the pin in the coordinate systems $(r, \theta)$ is $\left(r_{n}, \theta_{n}\right)$, at the moment $t$, the rotational angle of the free end of the pin relative to the axis of the disc is $\psi_{n}(t)$, and the rotational angle of the free end of the pin relative to that of the disc is $\alpha_{n}$, so $\psi_{n}=\Omega t+\theta_{n}-$ $\alpha_{n}$. Rotational effect and the effect of friction follower force are neglected and the equation of motion of the disc in the coordinate systems $(r, \theta)$ is

$$
\begin{aligned}
\rho_{d} h_{d} & \frac{\partial^{2} w(r, \theta, t)}{\partial t^{2}}+D_{d} \nabla^{4} w(r, \theta, t)+D_{d}^{*} \nabla^{4} \frac{\partial w(r, \theta, t)}{\partial t} \\
= & \sum_{n=1}^{n_{1}}-\frac{1}{r_{n}}\left[\delta\left(r-r_{n}\right) \delta\left(\theta-\alpha_{n}\right) F_{N n}\right],
\end{aligned}
$$

where $\nabla^{4}$ is Laplace operator defined in polar coordinate as $\nabla^{4}=\left(\partial^{2} / \partial r^{2}+\partial / r \partial r+\partial^{2} / r^{2} \partial \theta^{2}\right)^{2}$ and $D_{d}$ is flexible rigidity of the disc as $D_{d}=E_{d} h_{d}^{3} / 12\left(1-v_{d}^{2}\right)$, where $\rho_{d}$ is the density of the disc, $h_{d}$ is the thickness of the disc, $v_{d}$ is Poisson's ratio of the disc, and $D_{d}^{*}$ is the Kelvin-type damping coefficient of the disc.

2.1.3. Modeling of the Flexible Pin. The transverse vibration of the pin in the coordinate systems $(x, y)$ can be written as

$$
\begin{gathered}
E_{p} I_{p} \frac{\partial^{4} u^{t}(x, t)}{\partial x^{4}}+\rho_{p} S_{p} \frac{\partial^{2} u^{t}(x, t)}{\partial t^{2}}+c_{t} I_{p} \frac{\partial^{5} u^{t}(x, t)}{\partial x^{4} \partial t} \\
=\sum_{n=1}^{n_{1}} \delta\left(x-l_{P}\right) \\
\cdot\left(-F_{N n} \sin \gamma+\mu_{n} F_{N n} \cos \gamma+F_{t n} \cos \gamma\right),
\end{gathered}
$$

where $E_{p}$ is Young's modulus of the pin, $\rho_{p}$ is the density of the pin, $S_{p}$ is the cross-sectional area of the pin, and $c_{t}$ is the Kelvin-type damping coefficient of the pin in the transverse direction.

The axial vibration of the pin in the coordinate systems $(x, y)$ can be written as

$$
\begin{gathered}
\rho_{p} S_{p} \frac{\partial^{2} u^{a}(x, t)}{\partial t^{2}}-E_{p} S_{p} \frac{\partial^{2} u^{a}(x, t)}{\partial x^{2}}-c_{a} S_{p} \frac{\partial^{3} u^{a}(x, t)}{\partial x^{2} \partial t} \\
=\sum_{n=1}^{n_{1}} \delta\left(x-l_{P}\right)\left(F_{N n} \cos \gamma+\mu_{n} F_{N n} \sin \gamma+F_{t n} \sin \gamma\right),
\end{gathered}
$$

where $c_{a}$ is the Kelvin-type damping coefficient of the pin in the axial direction.

2.1.4. Dynamics of the Whole Pin-on-Disc System. Matrix form of coupled vibration equation of pin-on-disc system is built using modal synthesis method, which can be written as

$$
\begin{aligned}
\ddot{\mathbf{y}}+ & j 2 \Omega \mathbf{C} \dot{\mathbf{y}}-\Omega^{2} \mathbf{D} \mathbf{y}+\mathbf{C}_{s} \dot{\mathbf{y}}+j \Omega \mathbf{C C}_{s} \mathbf{y}+\left[\omega^{2}\right] \mathbf{y} \\
& +\sum_{n=1}^{n_{1}} K_{N n} \mathbf{A}_{N n} \mathbf{y}+\sum_{n=1}^{n_{1}} \mu_{n} K_{N n} \mathbf{B}_{N n} \mathbf{y}+\sum_{n=1}^{n_{1}} \xi K_{N n} \mathbf{A}_{t n} \mathbf{y} \\
& =\sum_{n=1}^{n_{1}} \mathbf{F}_{n} .
\end{aligned}
$$

The formula derivation process is shown in Appendix A.

\subsection{Parameters and Simulation Setup of the Pin-on-Disc} System. The parameters used for the pin-on-disc system are given in Appendix B.

The problem of squeal is considered in a frequency range of $1-12 \mathrm{kHz}$ so that only the first 13 modes of the disc, the 1st, 2nd, and 3rd transverse mode of the pin, and the first axial mode of the pin are used.

\subsection{Illustration of the Pin-on-Disc Model considering a Homogeneous Normal Contact Stiffness Distribution and a Homogeneous Friction Coefficient Distribution}

2.3.1. Calculation of the Pin-on-Disc Model. Assume that friction contact parameters (normal contact stiffness and friction coefficient) on all contact points are homogeneous distribution. And contact parameters are assumed to be $K_{N 1}=K_{N 2}=\cdots=K_{N 100}=K_{\text {mean }}$ and $\mu_{1}=\mu_{2}=$ $\cdots=\mu_{100}=\mu_{\text {mean }}$, where $K_{\text {mean }}$ is the mean value of the normal contact stiffness and $\mu_{\text {mean }}$ is the mean value of friction coefficient. The range of the contact stiffness is usually $10^{7}-10^{9} \mathrm{~N} / \mathrm{m}$, and the integral contact stiffness is assumed to be $3.5 \times 10^{8} \mathrm{~N} / \mathrm{m}$; the integral contact stiffness is divided into 100 parts, so the mean value is $K_{\text {mean }}=3.5 \times 10^{6} \mathrm{~N} / \mathrm{m}$. So a plot of predicted frequency (i.e., imaginary parts of eigenvalues) as a function of $\mu_{\text {mean }}$ in a frequency range of $1-10 \mathrm{kHz}$ can be derived, as is shown in Figures 2(a) and 2(b). It can be seen that the natural frequency of the $(0,1)$ 


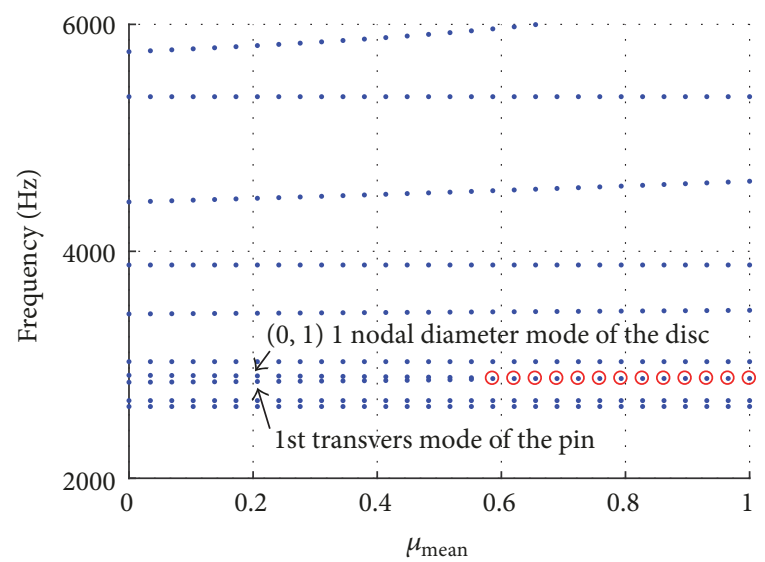

(a) In the frequency range of $2-6 \mathrm{kHz}$

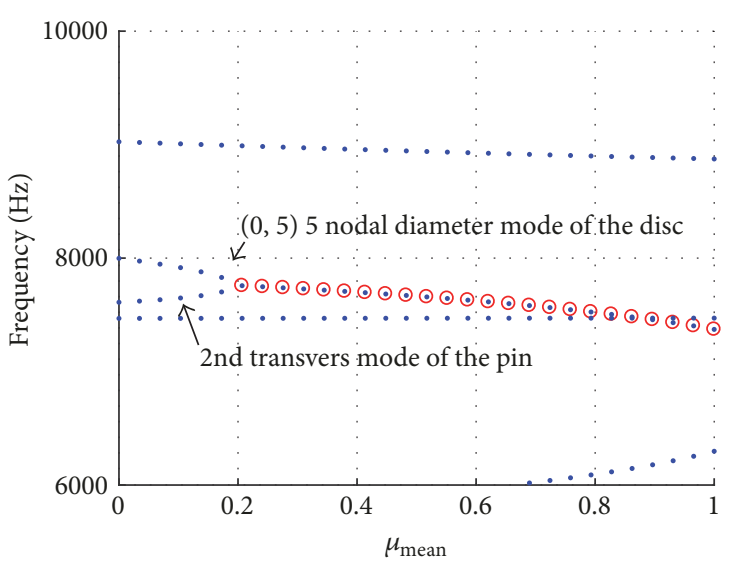

(b) In the frequency range of 6-10 kHz.

FIGURE 2: Evolution of the frequency versus $\mu_{\text {mean }}$.

nodal diameter mode of the disc combines with the natural frequency of the 1st transverse mode of the pin when the coefficient of friction is equal to 0.58 (call it the first unstable mode) and the natural frequency of the $(0,5)$ nodal diameter mode of the disc combines with the natural frequency of the 2nd transverse mode of the pin when the coefficient of friction is equal to 0.21 (call it the second unstable mode).

\subsubsection{The Region of Mode-Coupling Instability in Homoge-} neous Cases. A bounded region of mode-coupling instability of the first unstable mode and the second unstable mode for the normal contact stiffness versus friction coefficient is shown in Figures 3(a) and 3(b). It can be seen that the region of mode-coupling instability is now bounded in a finite normal contact stiffness and friction coefficient range. By selecting the proper normal contact stiffness and friction coefficient, the region of mode-coupling instability can be avoided.

\section{The Influence of Friction Contact Parameters with Heterogeneous Distributions on Frictional Squeal}

3.1. Analysis Process. The analysis process is shown in Figure 4. In this study, we analyze the influence of normal contact stiffness with heterogeneous distribution on frictional squeal when the friction coefficient is homogeneous (Case 1 ), the influence of friction coefficient with heterogeneous distribution on frictional squeal when the contact stiffness is homogeneous (Case 2), and the influence of normal contact stiffness and friction coefficient with heterogeneous distribution on frictional squeal (Case 3). To simplify this study, heterogeneous contact stiffness and friction coefficient obey normal distribution, respectively, and assume that the coefficient of dispersion of normal contact stiffness and the coefficient of dispersion of friction coefficient have the same value in Case 3 because the distributions of normal contact stiffness and friction coefficient are both related to surface topography. It is clear that, in a real configuration, the distributions of contact stiffness and friction coefficient probably cannot be represented by simple normal distributions. But this simple distribution enables us to understand the role of the heterogeneities of the contact stiffness and friction coefficient.

Now Case 1 is taken as an example to explain the analysis process as shown in Figure 4. In order to develop this analysis, a Monte Carlo simulation is performed by varying the distribution of normal contact stiffness and friction coefficient. (1) The mean value of the friction coefficient, the mean value of the normal contact stiffness, and the coefficient of dispersion of normal contact stiffness should be defined; then 100 values of contact stiffness obeying normal distribution are generated. Each value is given to one contact point; then a surface with normal distribution contact stiffness is generated. (2) 1000 surfaces with the same mean value of normal contact stiffness and the coefficient of dispersion of normal contact stiffness are obtained by using the method in step (1). (3) Complex eigenvalue analysis is carried out for each surface; then the influence of the mean value of normal contact stiffness and friction coefficient on the ratio of unstable cases (RUC) is analyzed, where RUC is the ratio of the number of the unstable cases to the total calculated cases (namely, 1000 calculations). (4) The influence of $K_{\text {mean }}$, $\mu_{\text {mean }}$, and $\varepsilon_{K}$ on RUC and the dispersions and distributions of frequencies and real parts of the complex eigenvalues are analyzed.

3.2. Generation of Heterogeneous Normal Contact Stiffness and Friction Coefficient. In this section, heterogeneous normal contact stiffness and friction coefficient are generated as follows (take the generation of heterogeneous normal contact stiffness as an example): the mean value of contact stiffness is $K_{\text {mean }}$ and the coefficient of dispersion of normal contact stiffness is $\varepsilon_{K}$, so the standard deviation is $\sigma_{K}=\varepsilon_{K} K_{\text {mean }}$; then the main range of the normal contact stiffness is [ $K_{\text {mean }}{ }^{-}$ $\left.3 \sigma_{K}, K_{\text {mean }}+3 \sigma_{K}\right]$; each value is given to one contact point and then a surface with normal distribution contact stiffness is generated. A surface with normal distribution contact stiffness with the mean value $K_{\text {mean }}=2.2 \times 10^{6} \mathrm{~N} / \mathrm{m}$ and the 


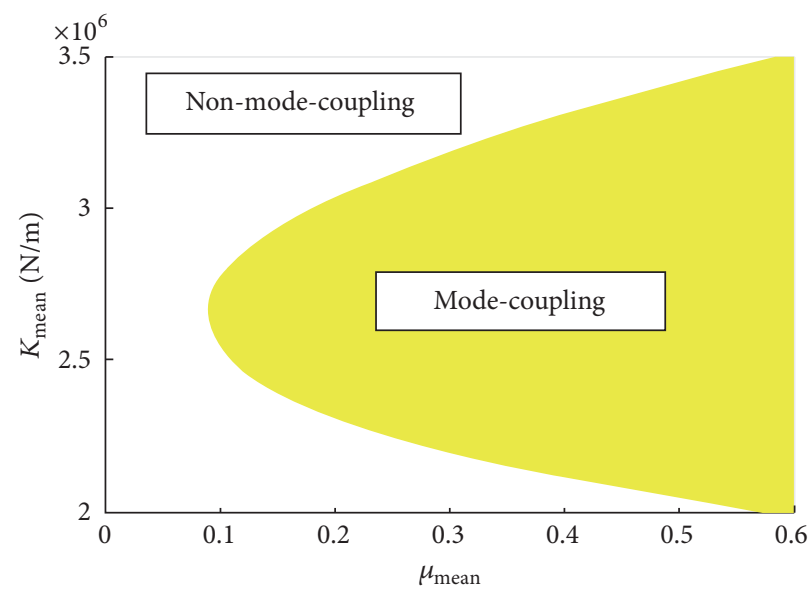

(a) The first unstable mode

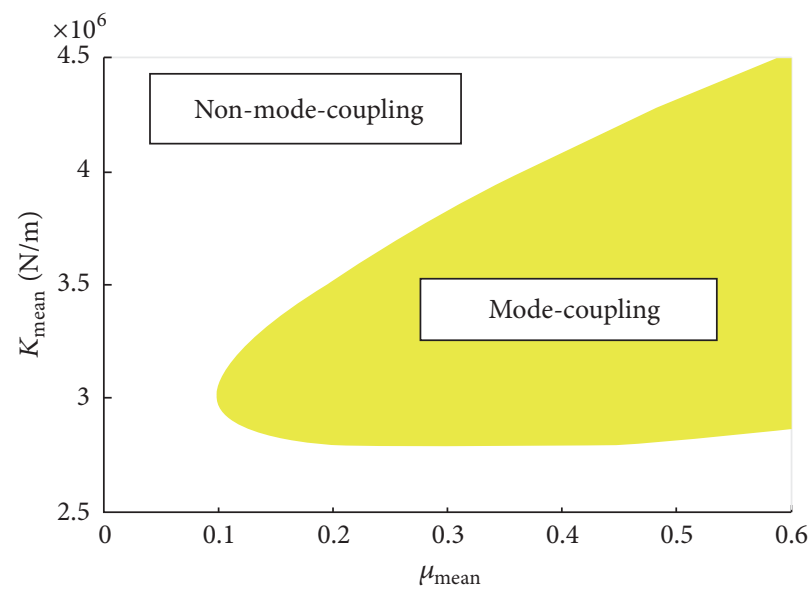

(b) The second unstable mode

FIGURE 3: The region of mode-coupling instability (normal contact stiffness and friction coefficient).

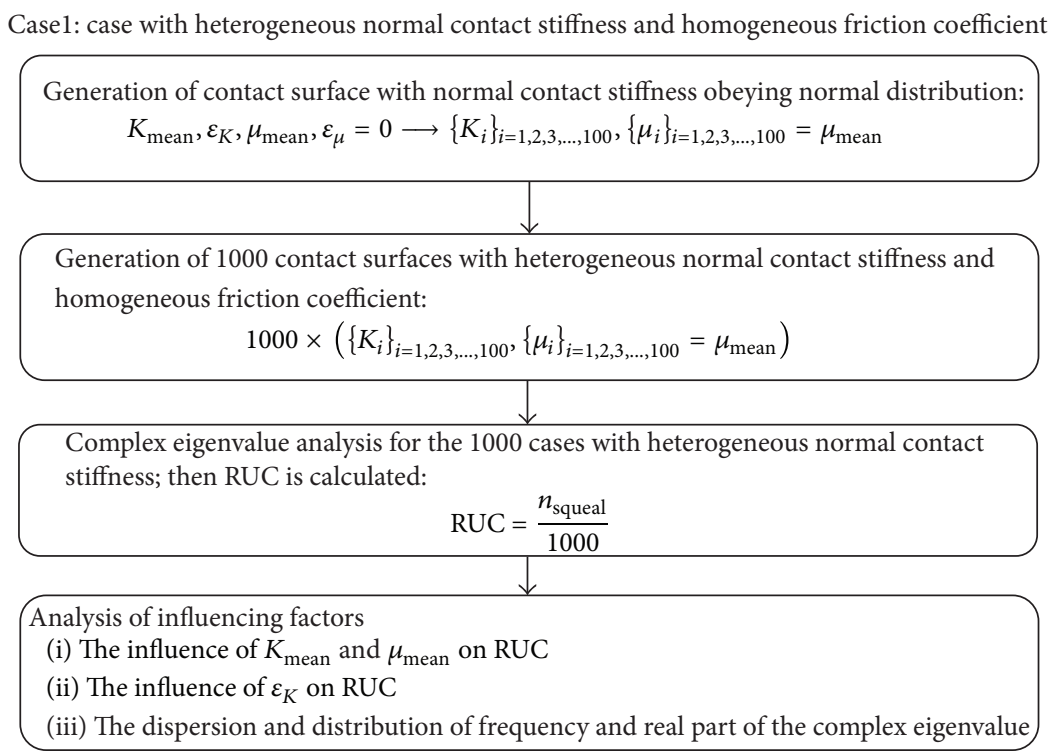

FIgURE 4: Analysis process.

coefficient of dispersion $\varepsilon_{K}=0.2$ is shown in Figures 5(a) and $5(\mathrm{~b})$.

\subsection{Evaluation Index of Heterogeneous Contact Stiffness and} Friction Coefficient. In this Section, 4 indexes are introduced, namely, the mean value of normal contact stiffness, the coefficient of dispersion of normal contact stiffness, the mean value of friction coefficient, and the coefficient of dispersion of friction coefficient. The mean values of the normal contact stiffness and friction coefficient indicate the magnitude of the normal contact stiffness and friction coefficient, and the coefficient of dispersions of the normal contact stiffness and friction coefficient indicate the degree of heterogeneity.

(1) The mean value of normal contact stiffness $K_{\text {mean }}$ is

$$
K_{\text {mean }}=\frac{\sum_{n=1}^{n_{1}} K_{N n}}{n_{1}} .
$$

(2) The coefficient of dispersion of normal contact stiffness $\varepsilon_{K}$ is

$$
\varepsilon_{K}=\frac{\sqrt{\left(1 / n_{1}\right) \sum_{n=1}^{n_{1}}\left(K_{n}-K_{\text {mean }}\right)^{2}}}{K_{\text {mean }}} .
$$

(3) The mean value of normal friction coefficient $\mu_{\text {mean }}$ is

$$
\mu_{\text {mean }}=\frac{\sum_{n=1}^{n_{1}} \mu_{n}}{n_{1}} .
$$

(4) The coefficient of dispersion of friction coefficient $\varepsilon_{\mu}$ is

$$
\varepsilon_{\mu}=\frac{\sqrt{\left(1 / n_{1}\right) \sum_{n=1}^{n_{1}}\left(\mu_{n}-\mu_{\text {mean }}\right)}}{\mu_{\text {mean }}} .
$$




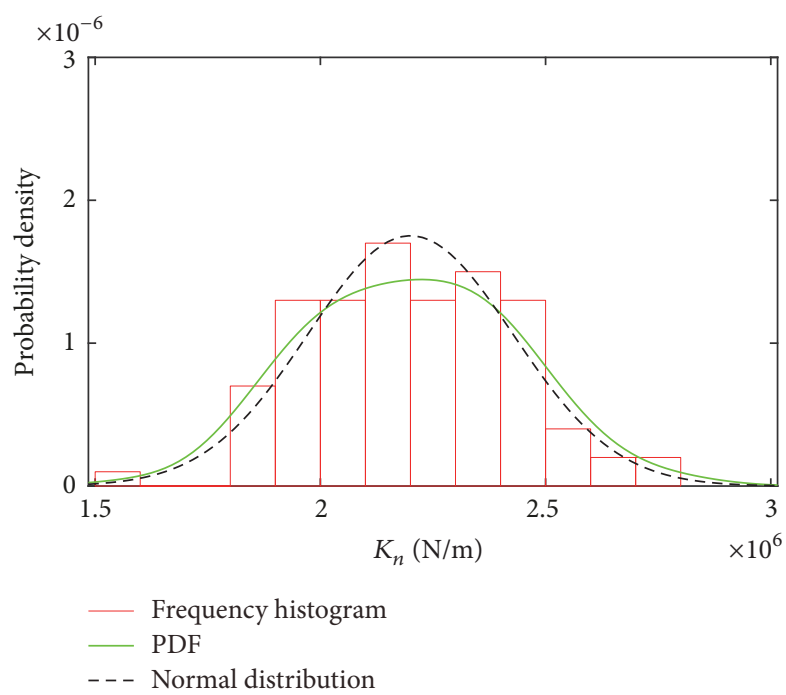

(a) Probability density of normal contact stiffness

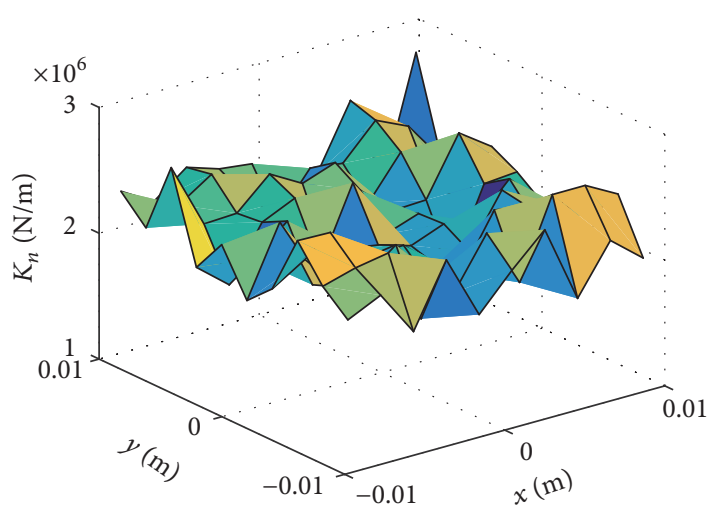

(b) Normal contact stiffness contact point

FIGURE 5: Surface with normal distribution contact stiffness.

3.4. Convergence Analysis. With the increasing of Monte Carlo simulations, the results would be more accurate, while the simulation time would increase greatly. In order to determine whether 1000 Monte Carlo simulations are sufficient to draw the conclusions, evolution of RUC versus the number of Monte Carlo simulations is analyzed. The analysis process is stated in Section 3.1, and the parameters used for the pin-ondisc system are given in Appendix B. Assume that $\mu_{\text {mean }}=$ $0.3, K_{\text {mean }}=2.2 \times 10^{6} \mathrm{~N} / \mathrm{m}$, and $\varepsilon_{K}=0.6$. Evolution of RUC versus the number of Monte Carlo simulations is shown in Figure 6. When the number of Monte Carlo simulations changes from 1000 to 11000 , RUC changes little. So, to save computation time, 1000 simulations are chosen. What is more, the computer (Dell-OptiPlex 3040, Intel core i5-6500) and the platform Matlab R2016b are used to run the Monte Carlo simulations.

\subsection{Case 1: Cases with Heterogeneous Normal Contact Stiffness and Homogeneous Friction Coefficient}

3.5.1. Influence of the Mean Value of Normal Contact Stiffness and Friction Coefficient on Frictional Squeal. The analysis process is stated in Section 3.1, and the parameters used for the pin-on-disc system are given in Appendix B. Assume that the coefficient of dispersion of normal contact stiffness is $\varepsilon_{K}=0.6$. The evolution of RUC versus the mean value of the normal contact stiffness and the mean value of the friction coefficient is shown in Figures 7(a) and 7(b), where the red arrow points to the enlarged image in the red square. In the enlarged image, the red line is the boundary of the region of mode-coupling instability when normal contact stiffness and friction coefficient are homogeneous distribution as shown in Figure 3.

(1) It shows that, to the first unstable mode and the second unstable mode, when $\left(\mu_{\text {mean }}, K_{\text {mean }}\right)$ locates out of the region of mode-coupling instability and far away from the boundary of the region of mode-coupling instability,

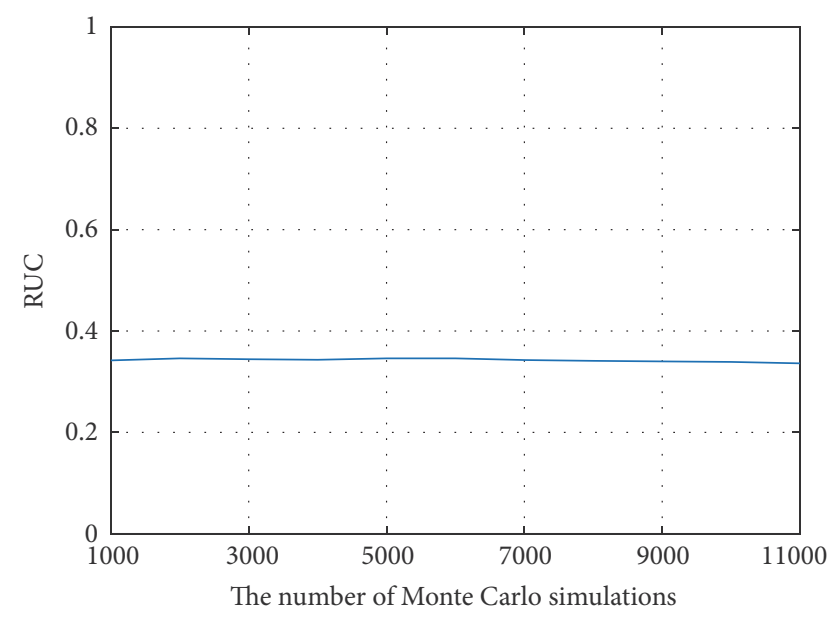

FIGURE 6: Evolution of RUC versus the number of Monte Carlo simulations.

RUC $=0$; when $\left(\mu_{\text {mean }}, K_{\text {mean }}\right)$ locates in the region of modecoupling instability and far away from the boundary of the region of mode-coupling instability, RUC $=1$; when $\left(\mu_{\text {mean }}, K_{\text {mean }}\right)$ locates near the boundary of the region of mode-coupling instability, $0<$ RUC $<1$.

(2) When $\left(\mu_{\text {mean }}, K_{\text {mean }}\right)$ locates near the boundary of the region of mode-coupling instability, the RUC increases with the increase of the mean value of normal contact stiffness and the mean value of friction coefficient.

(3) The result of complex eigenvalue analysis based on the model in homogeneous case should be only stable (RUC $=0$ ) or unstable $(\mathrm{RUC}=1)$, so in the region $0<\mathrm{RUC}<$ 1 , the model with homogeneous normal contact stiffness and friction coefficient cannot predict whether the system is stable or not. 


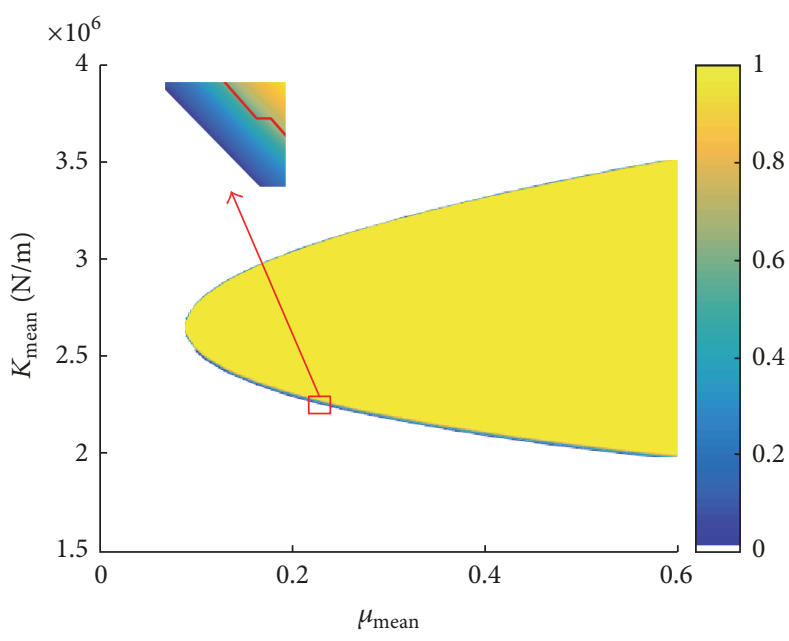

(a) The first unstable mode

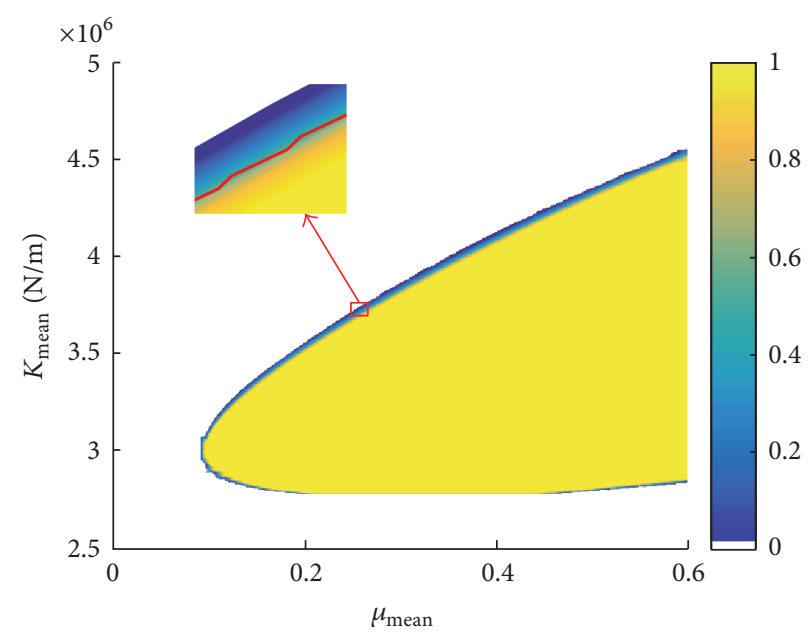

(b) The second unstable mode

Figure 7: Evolution of RUC versus the mean value of contact stiffness and the mean value of friction coefficient.

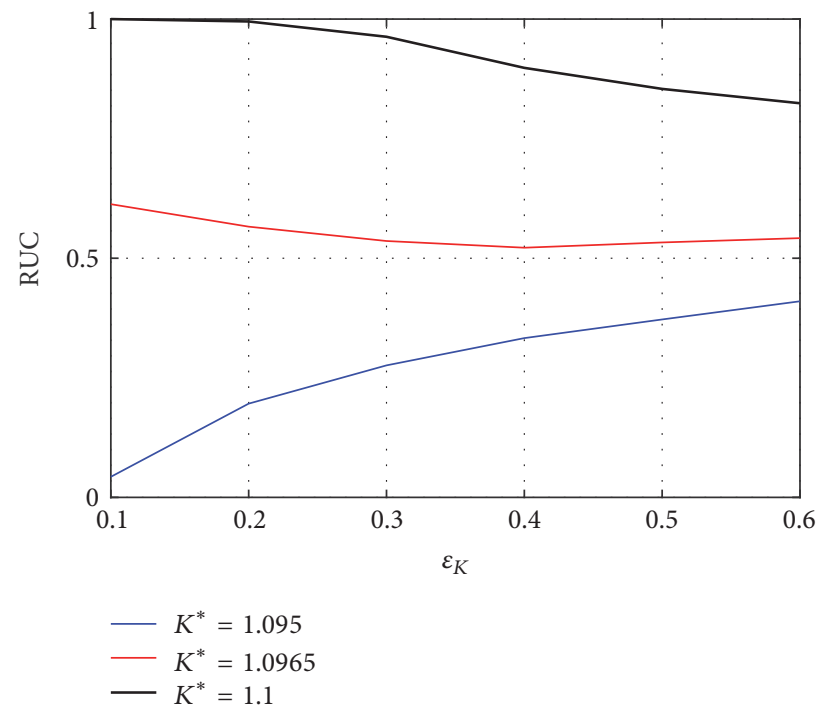

(a) The first unstable mode

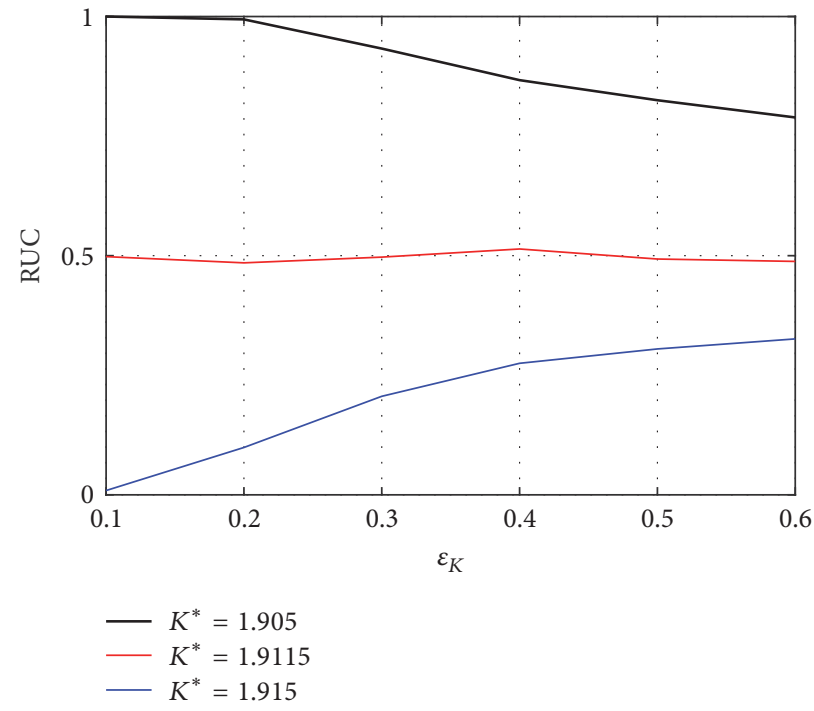

(b) The second unstable mode

FIGURE 8: Evolution of RUC versus coefficient of dispersion of normal contact stiffness.

3.5.2. Influence of the Coefficient of Dispersion of Normal Contact Stiffness on the Likelihood of Squeal Occurrence. Assuming that $\mu_{\text {mean }}=0.3$, variation of RUC with coefficient of dispersion of normal contact stiffness is analyzed when $\left(\mu_{\text {mean }}, K_{\text {mean }}\right)$ locates in the region of mode-coupling instability (the black line), in the non-mode-coupling region (the blue line), and on the boundary of region of mode-coupling instability (the red line), respectively, as shown in Figures 8(a) and 8 (b). Assuming that nominal value of normal contact stiffness is $K_{\text {nom }}=2 \times 10^{6} \mathrm{~N} / \mathrm{m}$, the dimensionless contact stiffness is $K^{*}=K_{\text {mean }} / K_{\text {nom }}$.

As shown in Figure 8, when $\left(\mu_{\text {mean }}, K_{\text {mean }}\right)$ locates in different position in Figure 3, RUC varies with the coefficient of dispersion of normal contact stiffness in different law: when $\left(\mu_{\text {mean }}, K_{\text {mean }}\right)$ locates in the non-mode-coupling region, RUC increases with the increase of the coefficient of dispersion of normal contact stiffness; when $\left(\mu_{\text {mean }}, K_{\text {mean }}\right)$ locates in the region of mode-coupling instability, RUC decreases with the increase of coefficient of dispersion of contact stiffness; when $\left(\mu_{\text {mean }}, K_{\text {mean }}\right)$ locates on the boundary of region of mode-coupling instability, RUC changes little with the increase of coefficient of dispersion of normal contact stiffness.

3.5.3. The Dispersions and Distributions of the Frequencies and Real Parts of the Complex Eigenvalues. The region in Figure 7 can be divided into 3 parts: stable region $(\mathrm{RUC}=0$ ), mix region $(0<$ RUC $<1)$, and unstable region $(R U C=1)$. In frictional squeal analysis, mix region and unstable region are more important. So frequencies and real parts of the complex eigenvalues in the mix region and the unstable region are analyzed. 
TABLE 1: Dispersions of frequencies and real parts of the complex eigenvalues of the first and the second unstable modes in the mix region.

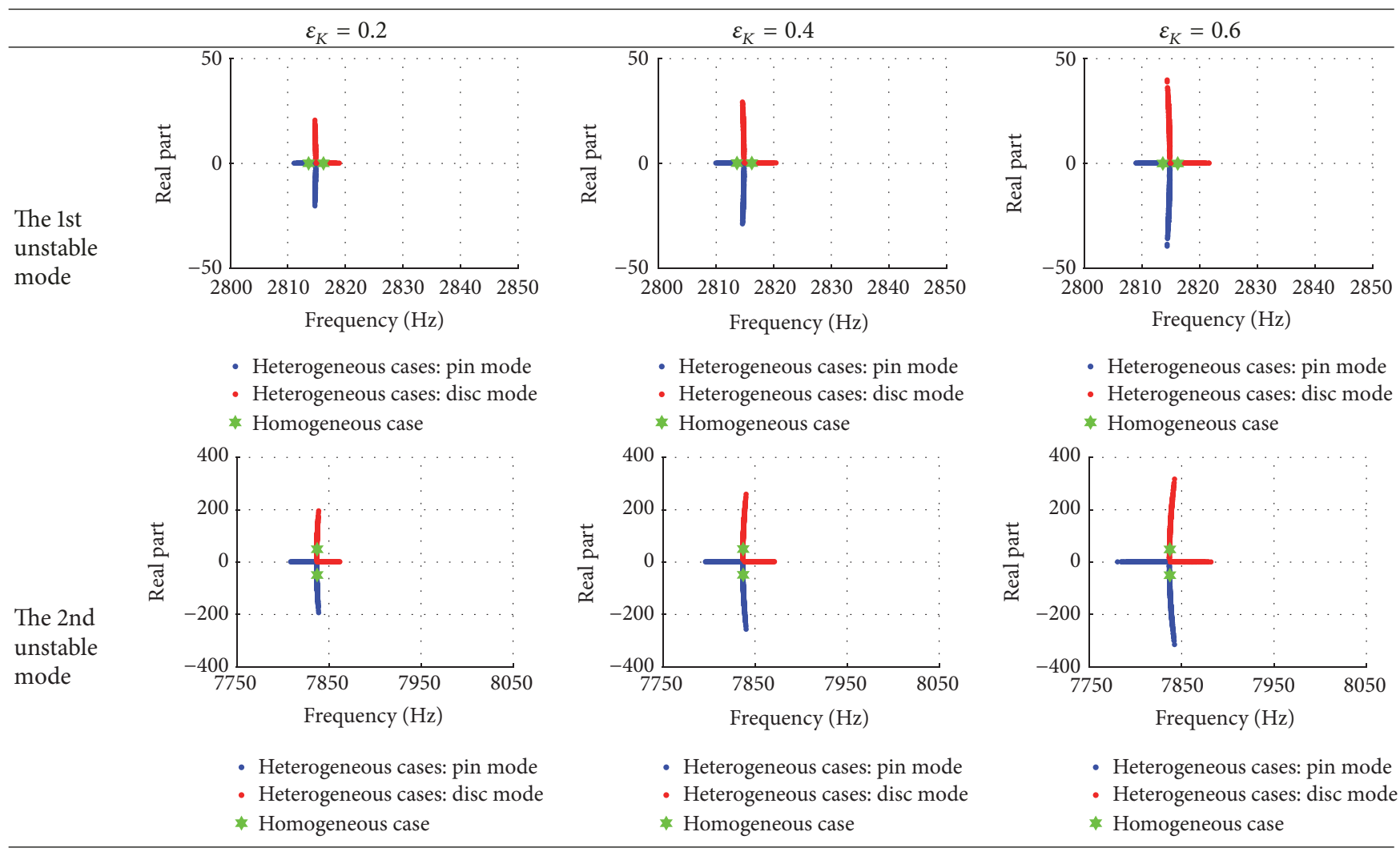

(1) The Dispersions of Frequencies and Real Parts of the Complex Eigenvalues in the Mix Region Selecting the point in the mix region $\left(\mu_{\text {mean }}=0.3, K_{\text {mean }}=2.19 \times 10^{6} \mathrm{~N} / \mathrm{m}\right.$ for the first unstable mode and $\mu_{\text {mean }}=0.3, K_{\text {mean }}=$ $3.825 \times 10^{6} \mathrm{~N} / \mathrm{m}$ for the second unstable mode), 1000 random surfaces are generated and complex eigenvalue analysis is carried out for $\varepsilon_{K}=0.2, \varepsilon_{K}=0.4, \varepsilon_{K}=0.6$, respectively. Then the dispersions of the frequencies and the real parts of the complex eigenvalues are obtained as shown in Table 1.

As shown in Table 1, the range of the frequencies and real parts of the complex eigenvalues changes around the frequency and real part of the complex eigenvalue in homogeneous case. And with the increase of the coefficient of dispersion of the normal contact stiffness, the range of the frequencies and the real parts of the complex eigenvalues of the first and the second unstable modes also increases.

(2) The Dispersions and Probability Densities of Frequencies and Real Parts of the Complex Eigenvalues in the Unstable Region Selecting the mean value of friction coefficient and contact stiffness in the unstable region $\left(\mu_{\text {mean }}\right.$ $=0.3, K_{\text {mean }}=2.5 \times 10^{6} \mathrm{~N} / \mathrm{m}$ for the first unstable mode and $\mu_{\text {mean }}=0.3, K_{\text {mean }}=4.2 \times 10^{6} \mathrm{~N} / \mathrm{m}$ for the second unstable mode), the dispersions and probability densities (PD) of frequencies and real parts of the complex eigenvalues in the unstable region are shown in Table 2, where the frequency histogram, the probability density function (PDF), and normal distribution with the same mean value and standard deviation are shown in the PD of frequency and real part of the complex eigenvalue. As shown in Table 2, with the increase of the coefficient of dispersion of the normal contact stiffness, the range of the frequencies and the real parts of the complex eigenvalues of the first and the second unstable modes also increases. And in the probability densities of the frequencies and real parts of the complex eigenvalues in Table 2, the PDFs of the frequencies and real parts of the complex eigenvalues approximate the normal distribution with the same mean value and standard deviation, which proves that the unstable frequencies and real parts of the complex eigenvalues of the first and the second unstable modes have normal distribution. The mean values of the frequencies of the first unstable mode when $\varepsilon_{K}=0.2, \varepsilon_{K}=0.4$, and $\varepsilon_{K}=0.6$ are $2835.47,2835.47$, and 2835.47 and the standard deviations are $0.04386,0.08299$, and 0.1267 , respectively. It is proven that, with the increase of the coefficient of dispersion of the normal contact stiffness, the mean values of frequencies of the first unstable mode are the same and the standard deviations of the contact stiffness increase. The mean values and the standard deviations of frequencies of the second unstable mode obey the same law as that of the first unstable mode, so unnecessary details are not given here.

\subsection{Case 2: Cases with Heterogeneous Friction Coefficient and Homogeneous Normal Contact Stiffness}

3.6.1. Influence of the Mean Value of Normal Contact Stiffness and Friction Coefficient on Frictional Squeal. The analysis process is stated in Section 3.1, and the parameters used for the pin-on-disc system are given in Appendix B. Assume that 


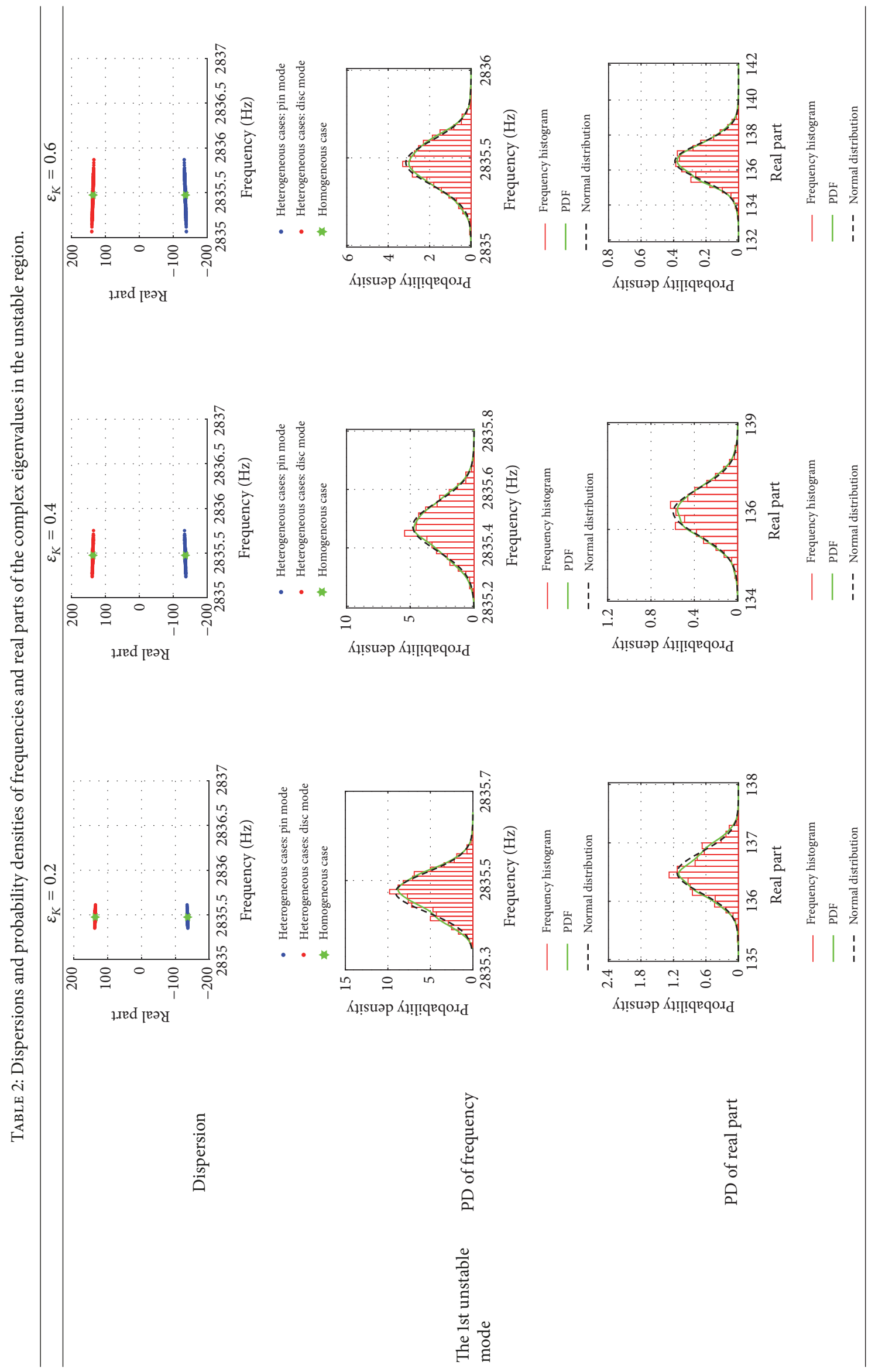




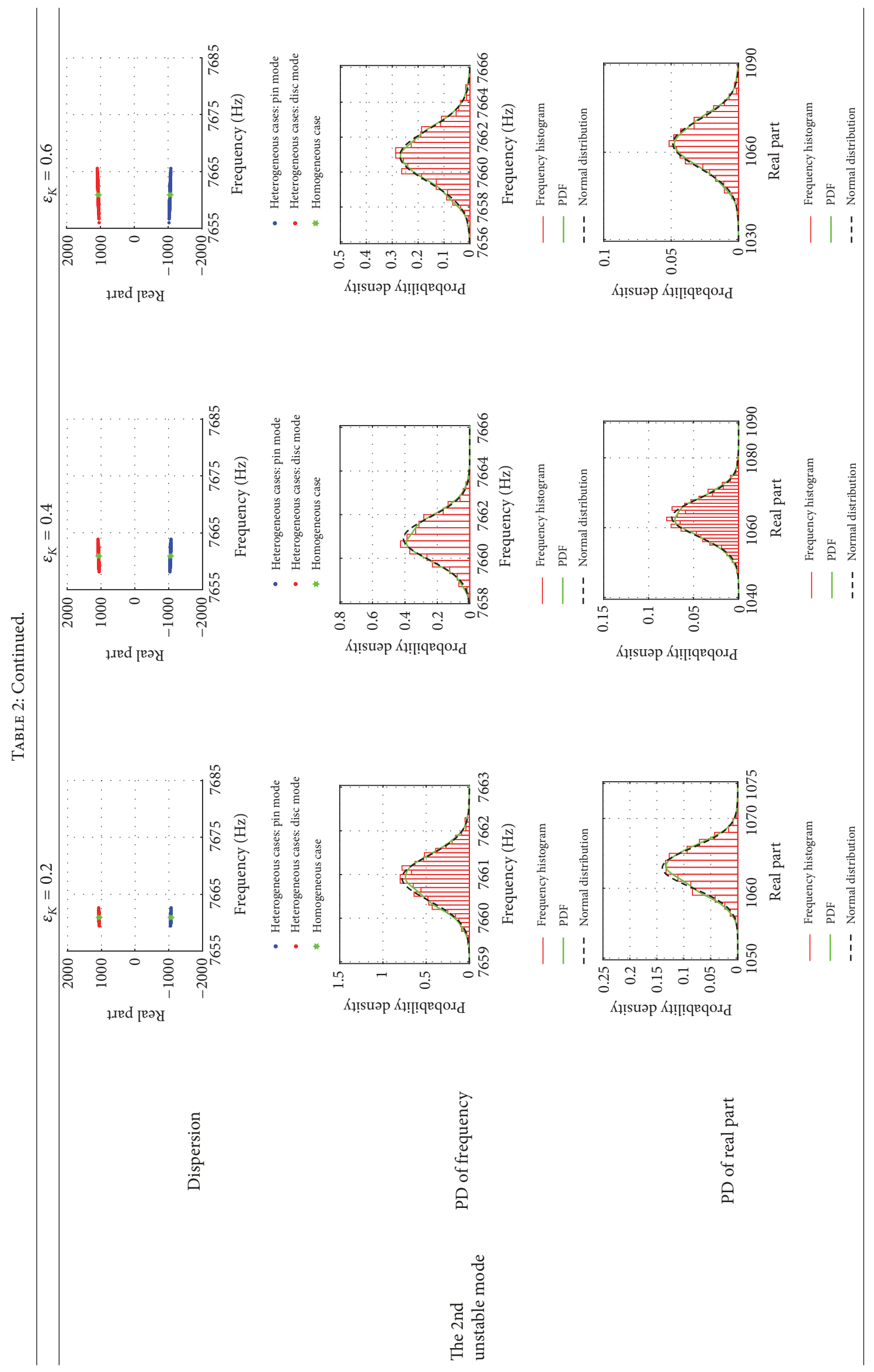


the coefficient of dispersion of friction coefficient is $\varepsilon_{\mu}=$ 0.6. The evolution of RUC versus the mean value of the normal contact stiffness and the mean value of the friction coefficient is shown in the second row in Table 5 (the row of Case 2), where the arrow points to the enlarged image in the red square. In the enlarged image, the red line indicates the boundary of the region of mode-coupling instability when normal contact stiffness and friction coefficient have homogeneous distribution as shown in Figure 3.

(1) It is shown that, to the first unstable mode and the second unstable mode, when $\left(\mu_{\text {mean }}, K_{\text {mean }}\right)$ locates out of the region of mode-coupling instability and far away from the boundary of the region of mode-coupling instability, RUC = 0 ; when $\left(\mu_{\text {mean }}, K_{\text {mean }}\right)$ locates in region of mode-coupling instability and far away from the boundary of the region of mode-coupling instability, RUC $=1$; when $\left(\mu_{\text {mean }}, K_{\text {mean }}\right)$ locates near the boundary of the region of mode-coupling instability, $0<$ RUC $<1$.

(2) When $\left(\mu_{\text {mean }}, K_{\text {mean }}\right)$ locates near the boundary of the region of mode-coupling instability, RUC increases with the increase of the mean value of normal contact stiffness and the mean value of the friction coefficient.

(3) The result of complex eigenvalue analysis based on the model with homogeneous cases should be only stable $($ RUC $=0)$ or unstable $($ RUC $=1)$, so in the region $0<$ RUC $<1$, the model with homogeneous normal contact stiffness and friction coefficient cannot predict whether the system is stable or not.

3.6.2. Influence of the Coefficient of Dispersion of Friction Coefficient on the Likelihood of Squeal Occurrence. As shown in the second row in Table 6 (the row of Case 2), RUC varies with the coefficient of dispersion of friction coefficient in different law: when $\left(\mu_{\text {mean }}, K_{\text {mean }}\right)$ locates in the non-mode-coupling region, RUC increases with the increase of coefficient of dispersion of friction coefficient; when $\left(\mu_{\text {mean }}, K_{\text {mean }}\right)$ locates in the region of mode-coupling instability, RUC decreases with the increase of the coefficient of dispersion of friction coefficient; when $\left(\mu_{\text {mean }}, K_{\text {mean }}\right)$ locates on the boundary of region of mode-coupling instability, RUC changes little with the increase of coefficient of dispersion of friction coefficient.

3.6.3. The Dispersions and Distributions of the Frequencies and Real Parts of the Complex Eigenvalues. Just like in Section 3.5.3, frequencies and real parts of the complex eigenvalues in the mix region and the unstable region are analyzed below.

(1) The Dispersions of Frequencies and Real Parts of the Complex Eigenvalues in the Mix Region Selecting the point in the mix region $\left(\mu_{\text {mean }}=0.3, K_{\text {mean }}=2.19 \times 10^{6} \mathrm{~N} / \mathrm{m}\right.$ for the first unstable mode and $\mu_{\text {mean }}=0.3, K_{\text {mean }}=3.825 \times 10^{6} \mathrm{~N} / \mathrm{m}$ for the second unstable mode), 1000 random surfaces are generated and complex eigenvalue analysis are carried out for $\varepsilon_{\mu}=0.2, \varepsilon_{\mu}=0.4, \varepsilon_{\mu}=0.6$, respectively. Then the dispersions of the frequencies and the real parts of the complex eigenvalues are obtained as shown in Table 7.

As shown in Table 7, with the increase of the coefficient of dispersion of the friction coefficient, the range of the frequencies and the real parts of the complex eigenvalues of the first and the second unstable modes increases.

(2) The Dispersions and Probability Densities of Frequencies and Real Parts of the Complex Eigenvalues in the Unstable Region Selecting the mean value of friction coefficient and contact stiffness in the unstable region $\left(\mu_{\text {mean }}\right.$ $=0.3, K_{\text {mean }}=2.5 \times 10^{6} \mathrm{~N} / \mathrm{m}$ for the first unstable mode and $\mu_{\text {mean }}=0.3, K_{\text {mean }}=4.2 \times 10^{6} \mathrm{~N} / \mathrm{m}$ for the second unstable mode), the dispersions and probability densities of frequencies and real parts of the complex eigenvalues in the unstable region are shown in Table 8, where the frequency histogram, the probability density function (PDF), and normal distribution with the same mean value and standard deviation are shown in the figures of distributions of frequencies and real parts of the complex eigenvalues. With the increase of the coefficient of dispersion of friction coefficient, the range of the frequencies and the real parts of the complex eigenvalues of the first and the second unstable modes also increases. And in the probability densities of the frequencies and real parts of the complex eigenvalues in Table 8, the PDFs of the frequencies and real parts of the complex eigenvalues approximate the normal distribution with the same mean value and standard deviation, which proves that the unstable frequencies and real parts of the complex eigenvalues are normally distributed. The mean values of the frequency of the first unstable mode when $\varepsilon_{\mu}=$ $0.2, \varepsilon_{\mu}=0.4$, and $\varepsilon_{\mu}=0.6$ are $2835.48,2835.48$, and 2835.48 and the standard deviations are $0.03341,0.06278$, and 0.9598 , respectively, which proves that the mean values of frequency of the first unstable mode are the same, and the standard deviations of the frequency increase with the increase of the coefficient of dispersion of friction coefficient. The mean values and the standard deviation of frequency of the second unstable mode obey the same law as that of the first unstable mode, so unnecessary details are not given here.

3.7. Case 3: Cases with Heterogeneous Normal Contact Stiffness and Heterogeneous Friction Coefficient. Under the condition that normal contact stiffness and friction coefficient have normal distribution, the evolution of RUC versus the mean value of the normal contact stiffness and the mean value of the friction coefficient is shown in the third row in Table 5 (the row of Case 3), and the evolution of RUC with the coefficient of dispersion of the contact stiffness $\left(\varepsilon_{K}=\varepsilon_{\mu}=\right.$ 0.6 ) is shown in the third row in Table 6 (the row of Case 3). The dispersions of the frequencies and real parts of the complex eigenvalues in the mix region are shown in Table 9 and the dispersions and distributions of the frequencies and real parts of the complex eigenvalues are shown in Table 10. Influence of $\left(\mu_{\text {mean }}, K_{\text {mean }}\right)$ on RUC and influence of the coefficient of dispersion of the contact stiffness and friction coefficient on RUC are the same as those in Case 1 and Case 2 ; at the same time, influences of the coefficient of dispersion of normal contact stiffness and friction coefficient on the frequencies and real parts of the complex eigenvalues in the mix region and the unstable region are the same as those in Case 1 and Case 2, and frequencies and real parts of the complex eigenvalues in unstable region also have normal distribution, so unnecessary details are not given here. 
Combining figures in the third row in Table 5 and Figure 7, it can be concluded that when contact stiffness and friction coefficient are both heterogeneous in Case 3, for the first and the second unstable modes, the mix region is larger than that in Case 1 and Case 2.

\section{Conclusion}

This paper describes the development of a complex eigenvalue analysis of a flexible pin-on-disc system considering tangential contact stiffness. The study has included a heterogeneous distribution of contact stiffness and a heterogeneous distribution of friction coefficient to investigate the influence of the heterogeneous distribution of normal contact stiffness and friction coefficient on frictional squeal. The heterogeneous distribution is assumed as normal distribution. Different coefficient of dispersions and mean values of normal contact stiffness and friction coefficient have been examined and, for each, 1000 random heterogeneity dispersions are computed to reach a probabilistic study using Monte Carlo algorithm. The result demonstrates that the introduction of heterogeneities in the contact surface plays a key role in frictional squeal.

(1) When the mean value of normal contact stiffness and friction coefficient varies from the stable region to the unstable region, RUC increases from 0 to 1 . In contrast to the conditional region of instability (the region of mode-coupling instability), the range of the region when squeal may occur $(\mathrm{RUC}>0)$ is enlarged.

(2) The result of the pin-on-disc model with homogeneous normal contact stiffness and friction coefficient can be only RUC $=0$ or RUC $=1$, so when $\left(\mu_{\text {mean }}, K_{\text {mean }}\right)$ locates in the range of $0<$ RUC $<1$, the model with homogeneous normal contact stiffness and friction coefficient cannot predict the dynamic behavior of the system accurately.

(3) When $\left(\mu_{\text {mean }}, K_{\text {mean }}\right)$ locates in different position in non-mode-coupling region or the region of mode-coupling instability in the homogeneous cases, RUC varies with the coefficient of dispersion of normal contact stiffness in different law: when $\left(\mu_{\text {mean }}, K_{\text {mean }}\right)$ locates in the non-modecoupling region, RUC increases with the increase of the coefficient of dispersion of normal contact stiffness or friction coefficient; when $\left(\mu_{\text {mean }}, K_{\text {mean }}\right)$ locates in the region of mode-coupling instability, RUC decreases with the increase of coefficient of dispersion of contact stiffness or friction coefficient; when $\left(\mu_{\text {mean }}, K_{\text {mean }}\right)$ locates on the boundary of the region of mode-coupling instability, RUC changes little with the increase of coefficient of dispersion of normal contact stiffness or friction coefficient.

(4) The range of frequency and real part of the complex eigenvalue increases with the increase of coefficient of dispersion of normal contact stiffness or friction coefficient. When the mean value $\left(\mu_{\text {mean }}, K_{\text {mean }}\right)$ locates in the unstable region, if the distributions of the normal contact stiffness and friction coefficient are normal distributions, the frequency and real part of the complex eigenvalue have normal distributions. And when the coefficient of dispersion of contact stiffness or friction coefficient increases, the mean values of the frequency and real part of the complex eigenvalue vary little while the coefficients of dispersion of frequency and real part of the complex eigenvalue increase.

\section{Appendix}

\section{A. The Formula Derivation Process}

The transverse vibration of the disc can be expressed by the summation of its natural modes and modal coordinates as

$$
w(r, \theta, t)=\sum_{k=0}^{\infty} \sum_{m=-\infty}^{\infty} \Phi_{k m}(r, \theta) q_{k m}(t),
$$

where $\Phi_{k m}(r, \theta)=R_{k m}(r) e^{j m \theta}(k=0,1,2, \ldots ; m=$ $0, \pm 1, \pm 2, \ldots)$, which satisfy the orthonormality conditions:

$$
\begin{aligned}
& \rho_{p} h_{d} \iint_{S_{d}} \Phi_{k m} \bar{\Phi}_{k_{1} m_{1}} r \mathrm{~d} r \mathrm{~d} \theta=\delta_{k k_{1}} \delta_{m m_{1}}, \\
& D_{d} \iint_{S_{d}} \nabla^{4} \Phi_{k m} \bar{\Phi}_{k_{1} m_{1}} r \mathrm{~d} r \mathrm{~d} \theta=\omega_{k_{1} m_{1}}^{2} \delta_{k k_{1}} \delta_{m m_{1}},
\end{aligned}
$$

where $\iint_{C s}=\int_{0}^{2 \pi} \int_{r_{\text {in }}}^{r_{\text {out }}}$ and the bar over a symbol denotes the complex conjugation. $\omega_{k m}=\beta_{m, n}^{2} \sqrt{D_{d} / \rho_{d} h_{d}}$ is the natural frequency of the disc. $R_{k, m}(r)$ is the combination of Bessel functions given as follows:

$$
\begin{aligned}
R_{k, m}(r)= & A_{k, m} J_{m}\left(\beta_{k, m} r\right)+Q_{k, m} Y_{m}\left(\beta_{k, m} r\right) \\
& +B_{k, m} I_{m}\left(\beta_{k, m} r\right)+F_{k, m} K_{m}\left(\beta_{k, m} r\right),
\end{aligned}
$$

where $J_{m}\left(\beta_{k, m} r\right), Y_{m}\left(\beta_{k, m} r\right)$ are Bessel functions of the first and second kinds of order $m$ and $I_{m}\left(\beta_{k, m} r\right)$ and $K_{m}\left(\beta_{k, m} r\right)$ are modified Bessel functions of the first and second kinds of order $m . A_{k, m}, Q_{k, m}, B_{k, m}, F_{k, m}$, and $\beta_{k, m}$ are the constant coefficients and they can be determined from the boundary condition [23] (note that the mode shapes of the disc are in the form of nodal circles and nodal diameters denoted, respectively, by the subscripts $k$ and $m$; and $j=\sqrt{-1}$ )

Similarly, the transverse vibration of the pin can be expressed by the summation of its natural modes and modal coordinates as

$$
u^{t}(x, t)=\sum_{i=1}^{\infty} \varphi_{i}^{t}(x) p_{i}^{t}(t)
$$

which satisfy the orthonormality conditions:

$$
\begin{gathered}
\rho_{p} S_{p} \int_{0}^{l_{P}} \varphi_{i}^{t}(x) \varphi_{s}^{t}(x) \mathrm{d} x=\delta_{i s}, \\
E_{p} I_{p} \int_{0}^{l_{P}} \frac{\mathrm{d}^{4} \varphi_{i}^{t}(x)}{\mathrm{d} x^{4}} \varphi_{s}^{t}(x) \mathrm{d} x=\omega_{i}^{t^{2}} \delta_{i s},
\end{gathered}
$$

where $\omega_{i}^{t}=v_{i}^{2} \sqrt{E_{P} I_{p} / \rho_{P} S_{p}}$ is the natural frequency of the transverse vibration of the pin and the mode shape of the transverse vibration of the pin can be expressed as

$$
\begin{aligned}
\varphi_{i}^{t}(x)= & A_{1 i} \sin v_{i} x+B_{1 i} \cos v_{i} x+C_{1 i} \operatorname{sh} v_{i} x \\
& +Q_{1 i} \operatorname{ch} v_{i} x,
\end{aligned}
$$


TABLE 3: Physical parameters used for the disc.

\begin{tabular}{lccccc}
\hline$r_{\text {in }} / \mathrm{mm}$ & $r_{\text {out }} / \mathrm{mm}$ & $h_{d} / \mathrm{mm}$ & $E_{d} / \mathrm{MPa}$ & $v_{d}$ & $\rho_{d} / \mathrm{kg} \cdot \mathrm{m}^{-3}$ \\
\hline 50 & 120 & 15 & 122000 & 0.23 & $7.19 \times 10^{3}$ \\
\hline
\end{tabular}

TABLE 4: Physical parameters used for the pin.

\begin{tabular}{lcccc}
\hline$l_{p} / \mathrm{mm}$ & $a_{p} / \mathrm{mm}$ & $E_{p} / \mathrm{MPa}$ & $\nu_{p}$ & $\rho_{p} / \mathrm{kg} \cdot \mathrm{m}^{-3}$ \\
\hline 160 & 20.5 & 85000 & 0.32 & $2.88 \times 10^{3}$ \\
\hline
\end{tabular}

TABLE 5: Variation of RUC with mean value of contact stiffness and mean value of friction coefficient.

Case 2 The first unstable mode

where $A_{1 i}, B_{1 i}, C_{1 i}, Q_{1 i}$, and $v_{i}$ are the constant values and they can be determined from the boundary condition.

The axial vibration of the pin can be expressed by the summation of its natural modes and modal coordinates as

$$
u^{a}(x, t)=\sum_{i=1}^{\infty} \varphi_{i}^{a}(x) p_{i}^{a}(t)
$$

which satisfy the orthonormality conditions:

$$
\begin{gathered}
\rho_{p} S_{p} \int_{0}^{l_{p}} \varphi_{i}^{a}(x) \varphi_{s}^{a}(x) \mathrm{d} x=\delta_{i s}, \\
-E_{p} S_{p} \int_{0}^{l_{p}} \frac{\mathrm{d}^{2} \varphi_{i}^{a}(x)}{\mathrm{d} x^{2}} \varphi_{s}^{a}(x) \mathrm{d} x=\omega_{i}^{a} \delta_{i s},
\end{gathered}
$$

where $\omega_{i}^{a}=v_{i}^{\prime} \sqrt{E_{p} / \rho_{p}}$ is the natural frequency of the axis vibration and the mode shape of the axial vibration of the pin can be expressed as

$$
\varphi_{i}^{a}(x)=A_{2 i} \sin \left(v_{i}^{\prime} x\right)+B_{2 i} \cos \left(v_{i}^{\prime} x\right),
$$

where $A_{2 i}, B_{2 i}$, and $v_{i}^{\prime}$ are the constant coefficients and they can be determined from the boundary condition.

With regard to the disc, substituting (A.1) into (5) in Section 2, multiplying (5) with $\bar{\Phi}_{r s}$, and then integrating with the help of (A.2) yield

$$
\begin{gathered}
q_{k_{1} m_{1}}^{\prime \prime}(t)+\frac{D_{d}^{*}}{D_{d}} \omega_{k_{1} m_{1}}^{2} q_{k_{1} m_{1}}^{\prime}(t)+\omega_{k_{1} m_{1}}^{2} q_{k_{1} m_{1}}(t) \\
=\sum_{n=1}^{n_{1}}\left[-F_{N n} R_{k_{1} m_{1}}\left(r_{n}\right) \exp \left(-j m_{1} \varphi_{n}\right)\right] .
\end{gathered}
$$


TABLE 6: Variation of RUC with coefficient of dispersion of contact stiffness and friction coefficient.

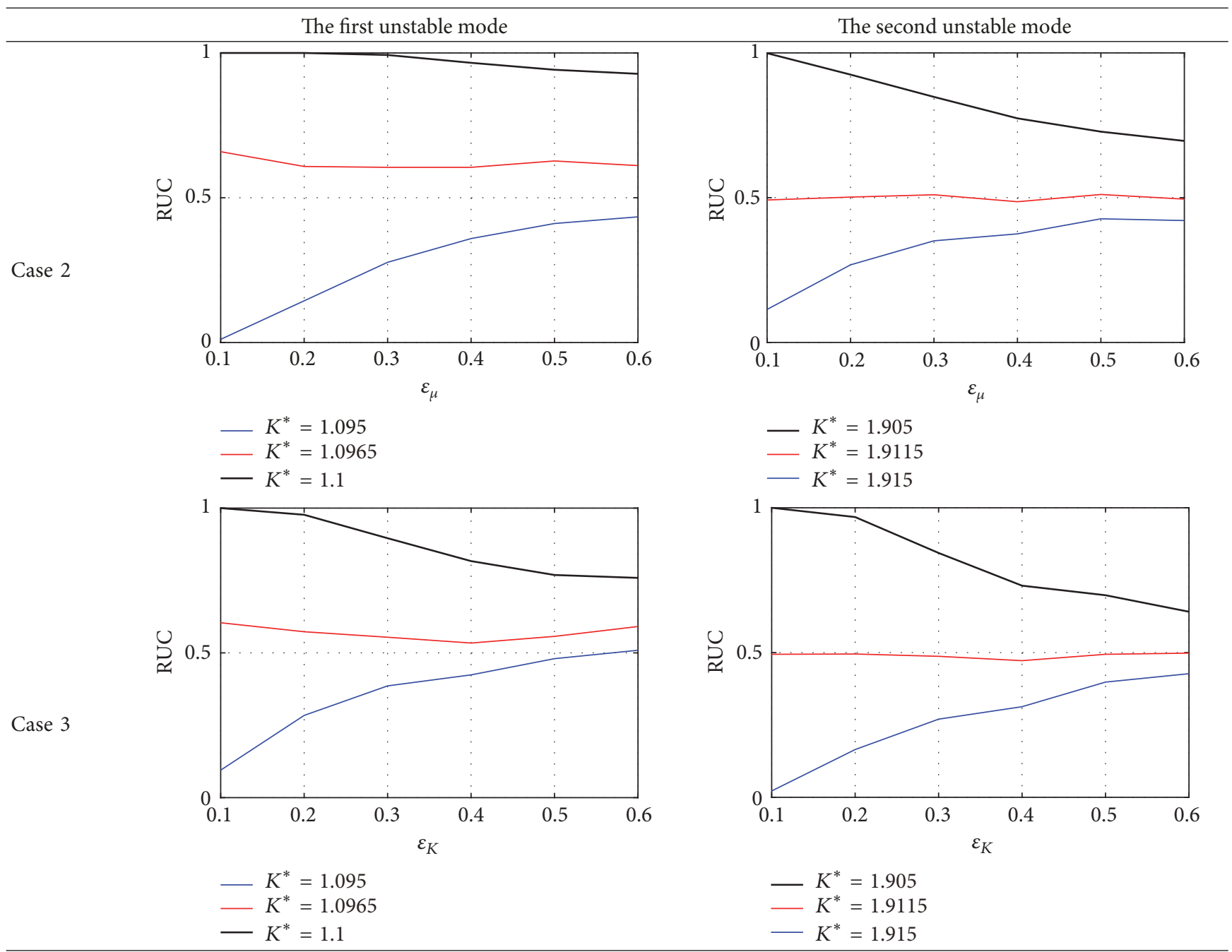

Similarly, with regard to the transverse vibration of the pin, substituting (A.4) into (6), multiplying (6) in Section 2 with $\varphi_{s}^{t}(x)$, and then integrating with the help of (A.5) yield

$$
\begin{aligned}
& p_{s}^{t^{\prime \prime}}(t)+\frac{c_{t}}{E_{p}} \omega_{s}^{t^{2}} p_{s}^{t^{\prime}}(t)+\omega_{s}^{t^{2}} p_{s}^{t}(t) \\
&= \sum_{n=1}^{n_{1}}\left(-F_{N n} \sin \gamma+\mu_{n} F_{N n} \cos \gamma+F_{t n} \cos \gamma\right) \\
& \cdot \varphi_{s}^{t}\left(l_{P}\right) .
\end{aligned}
$$

Similarly, with regard to the axial vibration of the pin, substituting (A.7) into (7) in Section 2, multiplying (7) with $\varphi_{s}^{a}(x)$, and then integrating with the help of (A.8) yield

$$
\begin{aligned}
& p_{s}^{a \prime \prime}(t)+\frac{c_{a}}{E_{p}} \omega_{s}^{a 2} p_{s}^{a \prime}(t)+\omega_{n}^{a 2} p_{s}^{a}(t) \\
& =\sum_{n=1}^{n_{1}}\left(F_{N n} \cos \gamma+\mu_{n} F_{N n} \sin \gamma+F_{t n} \sin \gamma\right) \varphi_{s}^{a}\left(l_{P}\right) .
\end{aligned}
$$

Considering that $\psi_{n}=\Omega t-\alpha_{n}+\theta_{n}=\left(u^{a}\left(l_{p}, t\right) \sin \gamma+\right.$ $\left.u^{t}\left(l_{p}, t\right) \cos \gamma\right) / r_{n}$ is marginal compared with $\phi_{n}$, for the sake of simplification, $\psi_{n}$ is neglected in the model so that

$$
\alpha_{n}=\Omega t+\theta_{n} .
$$

Substituting (A.1), (A.4), and (A.7) into (1) and (2) in Section 2 yields

$$
\begin{aligned}
F_{N n} & =K_{N n}\left(-u^{a}\left(l_{P}, t\right) \cos \gamma+u^{t}\left(l_{P}, t\right) \sin \gamma\right. \\
& \left.+w\left(r_{n}, \alpha_{n}, t\right)\right)+F_{L n} \\
& =K_{N n}\left(-\sum_{i=1}^{\infty} \varphi_{i}^{a}\left(l_{P}\right) p_{i}^{a}(t) \cos \gamma\right. \\
& +\sum_{i=1}^{\infty} \varphi_{i}^{t}\left(l_{P}\right) p_{i}^{t}(t) \sin \gamma \\
& \left.+\sum_{k=0}^{\infty} \sum_{l=-\infty}^{\infty} \Phi_{k l}\left(r_{n}, \alpha_{n}\right) q_{k l}(t)\right)+F_{L n}
\end{aligned}
$$


TABLE 7: Dispersions of frequencies and real parts of the complex eigenvalues of the first and the second unstable modes in the mix region in Case 2.

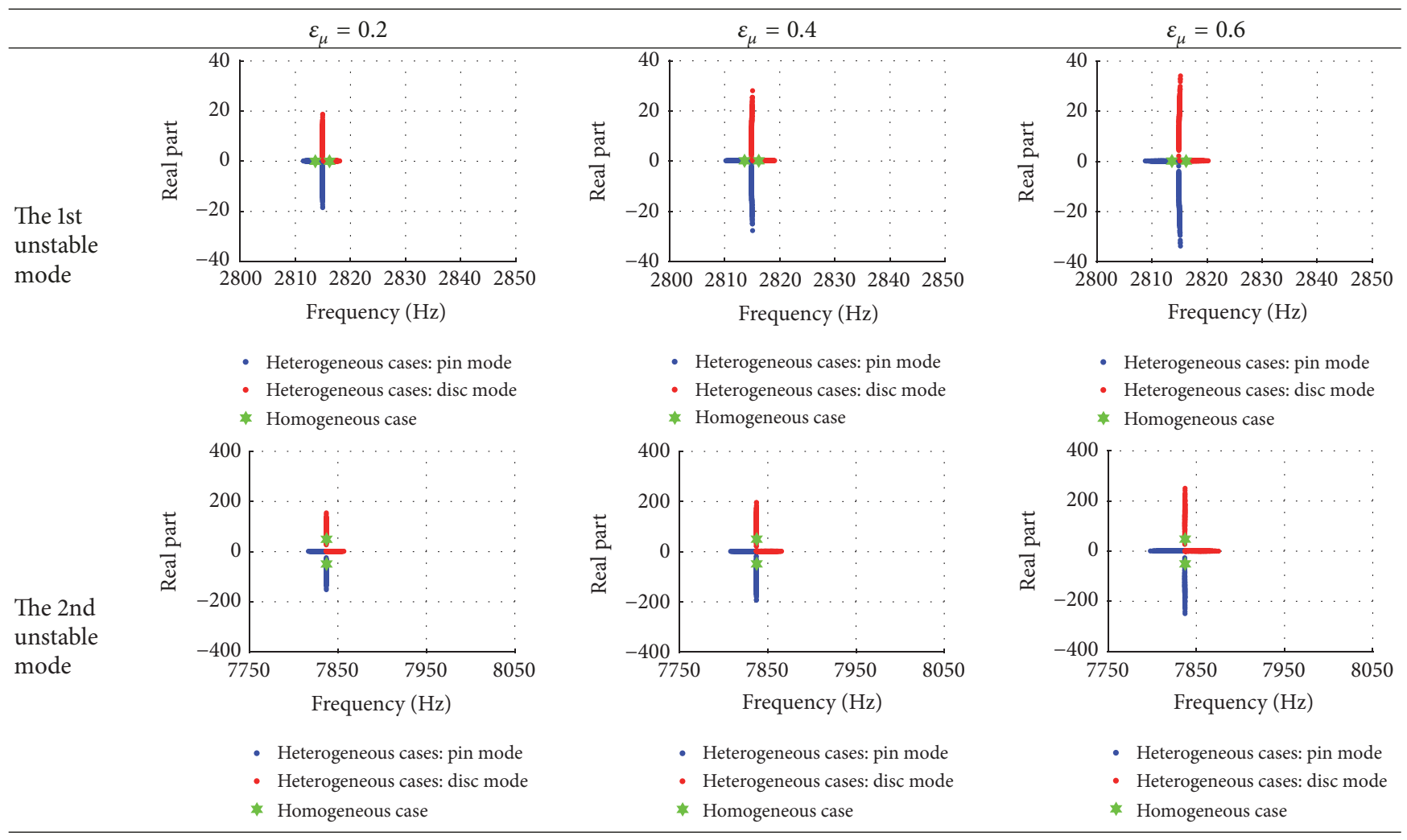

$$
\begin{aligned}
F_{t n} & =K_{t n}\left(u^{a}\left(l_{P}, t\right) \sin \gamma+u^{t}\left(l_{P}, t\right) \cos \gamma\right) \\
& =K_{t n}\left(\sum_{i=1}^{\infty} \varphi_{i}^{a}\left(l_{P}\right) p_{i}^{a}(t) \sin \gamma\right. \\
& \left.+\sum_{i=1}^{\infty} \varphi_{i}^{t}\left(l_{P}\right) p_{i}^{t}(t) \cos \gamma\right) .
\end{aligned}
$$

$$
\begin{aligned}
& +\sum_{i=1}^{\infty} \varphi_{i}^{t}\left(l_{P}\right) p_{i}^{t}(t) \sin \gamma \\
& \left.\left.+\sum_{k=0}^{\infty} \sum_{m=-\infty}^{\infty} R_{k m}\left(r_{n}\right) x_{k m}(t) e^{j m \theta_{n}}\right)+F_{L n}\right] \\
& \cdot R_{k_{1} m_{1}}\left(r_{n}\right) e^{-j m_{1} \theta_{n}}
\end{aligned}
$$

To make use of the method of state space, new modal coordinates are introduced as

$$
\begin{gathered}
x_{k_{1} m_{1}}(t)=q_{k_{1} m_{1}}(t) e^{j m_{1} \phi} \\
\left(k_{1}=0,1,2, \ldots ; m_{1}=0, \pm 1, \pm 2, \ldots\right), \\
p_{s}^{t}(t) \quad(s=1,2, \ldots), \\
p_{s}^{a}(t) \quad(s=1,2, \ldots) .
\end{gathered}
$$

Substituting (A.14), (A.15), (A.16), and (3) into (A.10), (A.11), and (A.12) yields

$$
\begin{aligned}
& x_{k_{1} m_{1}}^{\prime \prime}(t)-2 j m_{1} \phi^{\prime} x_{k_{1} m_{1}}^{\prime}(t)-m_{1}^{2} \phi^{\prime 2} x_{k_{1} m_{1}}(t) \\
& +\frac{D_{d}^{*}}{D_{d}} \omega_{k_{1} m_{1}}^{2}\left(x_{k_{1} m_{1}}^{\prime}(t)-j m_{1} \phi^{\prime} x_{k_{1} m_{1}}(t)\right) \\
& +\omega_{k_{1} m_{1}}^{2} x_{k_{1} m_{1}}(t) \\
& =-\sum_{n=1}^{n_{1}}\left[K _ { N n } \left(-\sum_{i=1}^{\infty} \varphi_{i}^{a}\left(l_{P}\right) p_{i}^{a}(t) \cos \gamma\right.\right.
\end{aligned}
$$

$$
\begin{aligned}
p_{s}^{t^{\prime \prime}}(t)+\frac{c_{t}}{E_{p}} \omega_{s}^{t^{2}} p_{s}^{t^{\prime}}(t)+\omega_{s}^{t^{2}} p_{s}^{t}(t) \\
=\sum_{n=1}^{n_{1}}\left[K _ { N n } \left(-\sum_{i=1}^{\infty} \varphi_{i}^{a}\left(l_{P}\right) p_{i}^{a}(t) \cos \gamma\right.\right. \\
+\sum_{i=1}^{\infty} \varphi_{i}^{t}\left(l_{P}\right) p_{i}^{t}(t) \sin \gamma \\
\left.\left.+\sum_{k=0}^{\infty} \sum_{m=-\infty}^{\infty} R_{k m}\left(r_{n}\right) x_{k m}(t) e^{j m \theta_{n}}\right)+F_{L n}\right] \\
\cdot\left(-\sin \gamma+\mu_{n} \cos \gamma\right) \varphi_{s}^{t}\left(l_{P}\right) \\
+\sum_{n=1}^{n_{1}}\left[K _ { t n } \left(-\sum_{i=1}^{\infty} \varphi_{i}^{a}\left(l_{P}\right) p_{i}^{a}(t) \sin \gamma\right.\right. \\
\left.\left.\quad-\sum_{i=1}^{\infty} \varphi_{i}^{t}\left(l_{P}\right) p_{i}^{t}(t) \cos \gamma\right)\right] \varphi_{s}^{t}\left(l_{P}\right) \cos \gamma,
\end{aligned}
$$




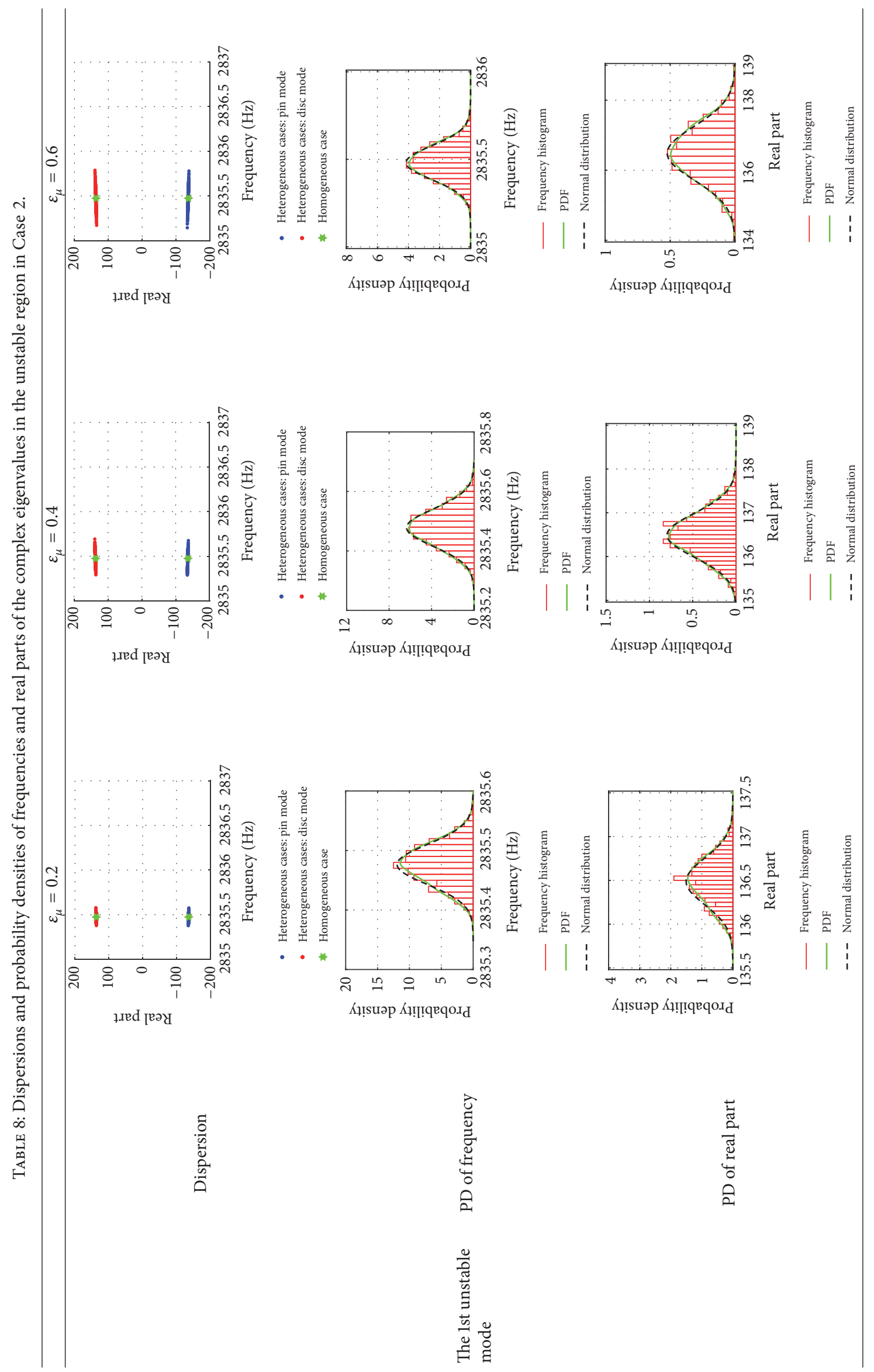




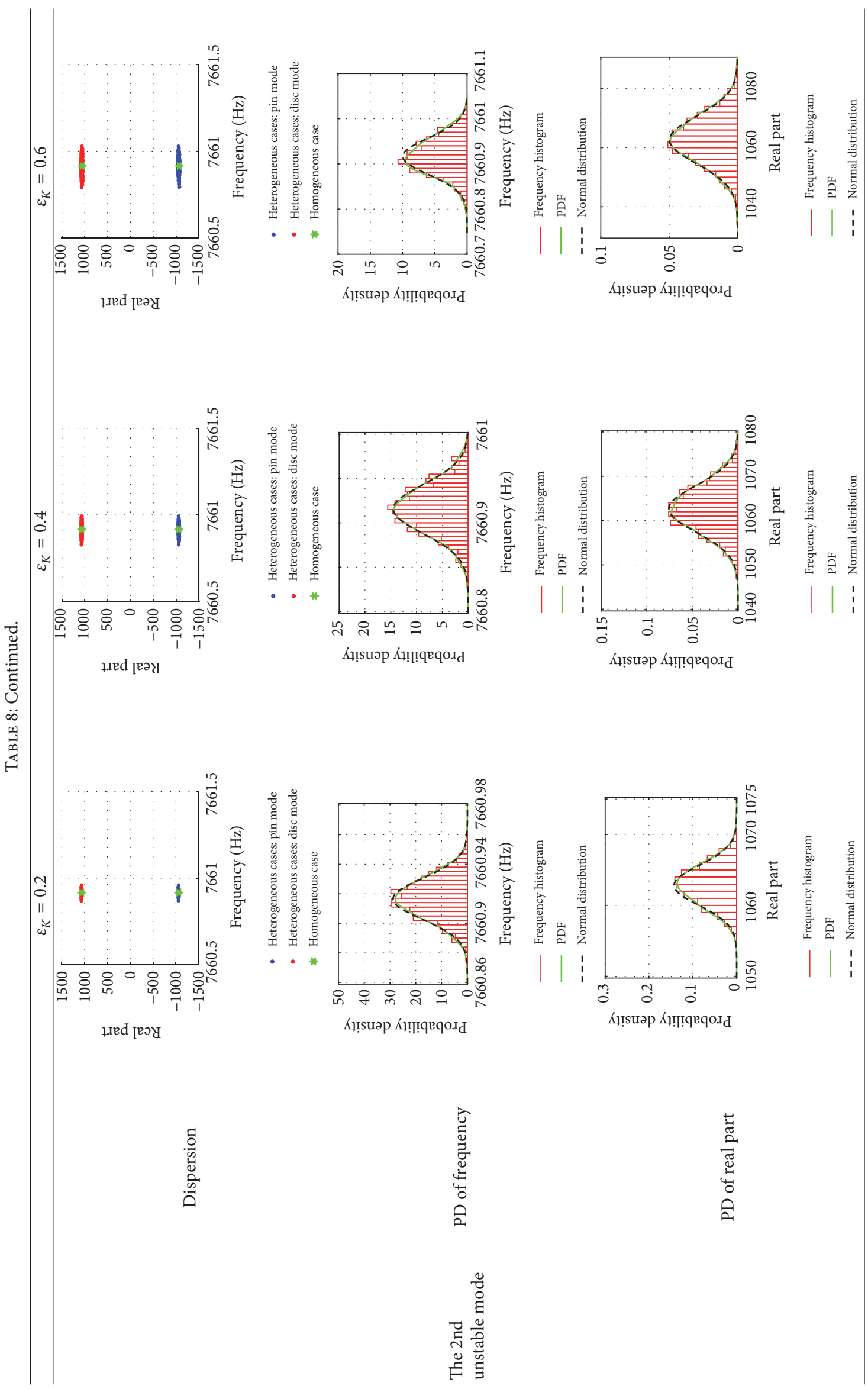


TABLE 9: Dispersions of frequencies and real parts of the complex eigenvalues of the first and the second unstable modes in the mix region in Case 3.

The 1 st unstable mode

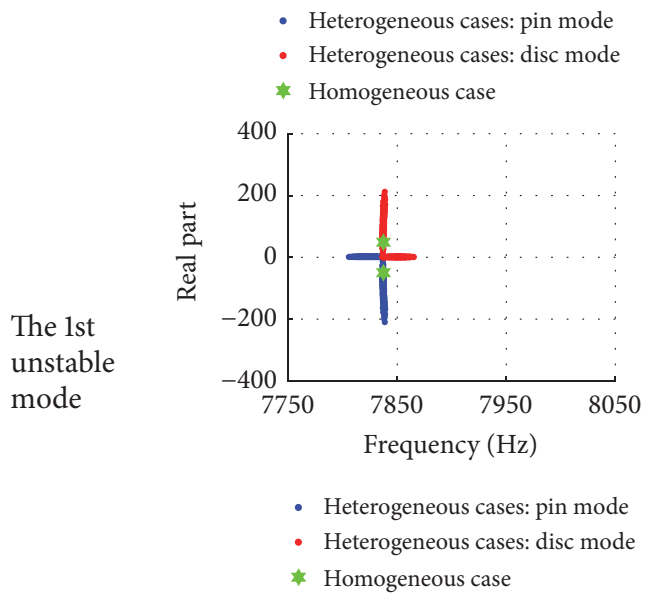

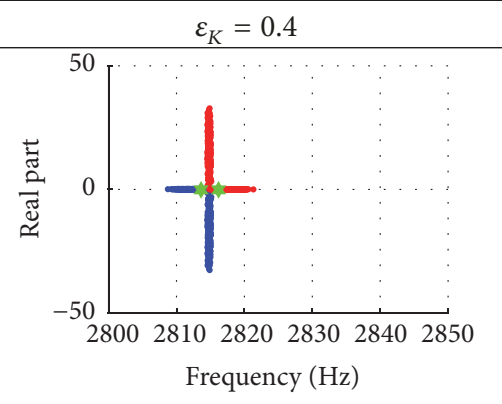

- Heterogeneous cases: pin mode

- Heterogeneous cases: disc mode

* Homogeneous case

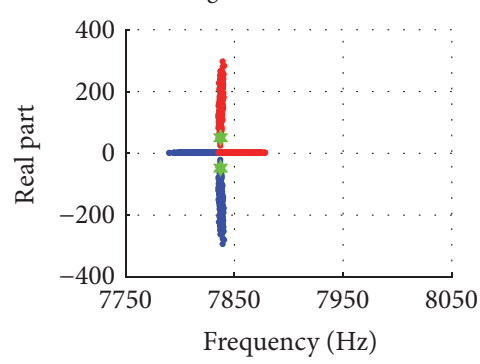

- Heterogeneous cases: pin mode

- Heterogeneous cases: disc mode

* Homogeneous case

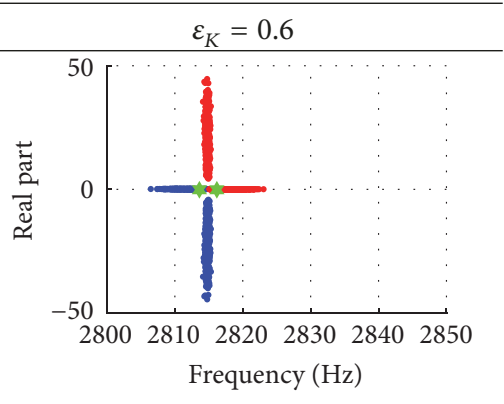

- Heterogeneous cases: pin mode

- Heterogeneous cases: disc mode

* Homogeneous case

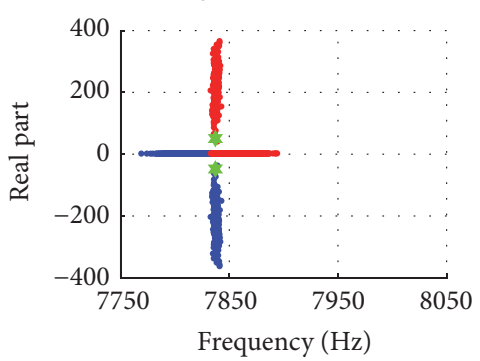

- Heterogeneous cases: pin mode

- Heterogeneous cases: disc mode

* Homogeneous case

$$
\begin{aligned}
p_{s}^{a \prime \prime} & (t)+\frac{c_{a}}{E} \omega_{s}^{a 2} p_{s}^{a \prime}(t)+\omega_{s}^{a 2} p_{s}^{a}(t) \\
& =\sum_{n=1}^{n_{1}}\left[K _ { N n } \left(-\sum_{i=1}^{\infty} \varphi_{i}^{a}\left(l_{P}\right) p_{i}^{a}(t) \cos \gamma\right.\right. \\
& +\sum_{i=1}^{\infty} \varphi_{i}^{t}\left(l_{P}\right) p_{i}^{t}(t) \sin \gamma \\
& \left.\left.+\sum_{k=0}^{\infty} \sum_{m=-\infty}^{\infty} R_{k m}\left(r_{n}\right) x_{k m}(t) e^{j m \theta_{n}}\right)+F_{L n}\right](\cos \gamma \\
+ & \left.\mu_{n} \sin \gamma\right) \varphi_{s}^{a}\left(l_{P}\right) \\
+ & \sum_{n=1}^{n_{1}}\left[K _ { t n } \left(-\sum_{i=1}^{\infty} \varphi_{i}^{a}\left(l_{P}\right) p_{i}^{a}(t) \sin \gamma\right.\right. \\
& \left.\left.-\sum_{i=1}^{\infty} \varphi_{i}^{t}\left(l_{P}\right) p_{i}^{t}(t) \cos \gamma\right)\right] \varphi_{s}^{a}\left(l_{P}\right) \sin \gamma .
\end{aligned}
$$

where $\xi_{k_{1} m_{1}}$ is the damping ratio of the vibration of the disc. $\xi_{s}^{t}$ is the damping ratio of the transverse vibration of the pin. $\xi_{s}^{a}$ is the damping ratio of the axial vibration of the pin.

Define

$$
\begin{aligned}
& \mathbf{y}=\left(\left\{x_{k m}(t)\right\}_{d},\left\{p_{i}^{t}(t)\right\}_{p}^{t},\left\{p_{i}^{a}(t)\right\}_{p}^{a}\right)^{T}, \\
& \mathbf{C}=-\operatorname{diag}\left[\{m\}_{d},\{0\}_{p}^{t},\{0\}_{p}^{a}\right], \\
& \mathbf{D}=\mathbf{C}^{2}, \\
& \mathbf{C}_{s}=2 \operatorname{diag}\left[\left\{\xi_{k m} \omega_{k m}\right\}_{d},\left\{\xi_{i}^{t} \omega_{i}^{t}\right\}_{p}^{t},\left\{\xi_{i}^{a} \omega_{i}^{a}\right\}_{p}^{a}\right] \\
& {\left[\omega^{2}\right]=\operatorname{diag}\left[\left\{\omega_{k m}^{2}\right\}_{d},\left\{\omega_{i}^{t^{2}}\right\}_{p}^{t},\left\{\omega_{i}^{a}\right\}_{p}^{a}\right]} \\
& \mathbf{R}_{A 1 n}=\left(\left\{R_{k m}\left(r_{n}\right) e^{j m \theta_{n}}\right\}_{d},\left\{\varphi_{i}^{t}\left(l_{P}\right) \sin \gamma\right\}_{p}^{t},\right. \\
& \left.\quad\left\{-\varphi_{i}^{a}\left(l_{P}\right) \cos \gamma\right\}_{p}^{a}\right), \\
& \mathbf{R}_{A 2 n}=\left(\left\{R_{k m}\left(r_{n}\right) e^{-j m \theta_{n}}\right\}_{d},\left\{\varphi_{i}^{t}\left(l_{P}\right) \sin \gamma\right\}_{p}^{t},\right. \\
& \left.\left\{-\varphi_{i}^{a}\left(l_{P}\right) \cos \gamma\right\}_{p}^{a}\right), \\
& \mathbf{R}_{C}=\left(\{0\}_{d},\left\{\varphi_{i}^{t}\left(l_{P}\right) \cos \gamma\right\}_{p}^{t},\left\{\varphi_{i}^{a}\left(l_{P}\right) \sin \gamma\right\}_{p}^{a}\right), \\
& \mathbf{A}_{N n}=\mathbf{R}_{A 2 n}^{T} \mathbf{R}_{A 1 n},
\end{aligned}
$$

$$
\frac{c_{a}}{E_{p}} \omega_{s}^{a 2}=2 \xi_{s}^{a} \omega_{s}^{a},
$$




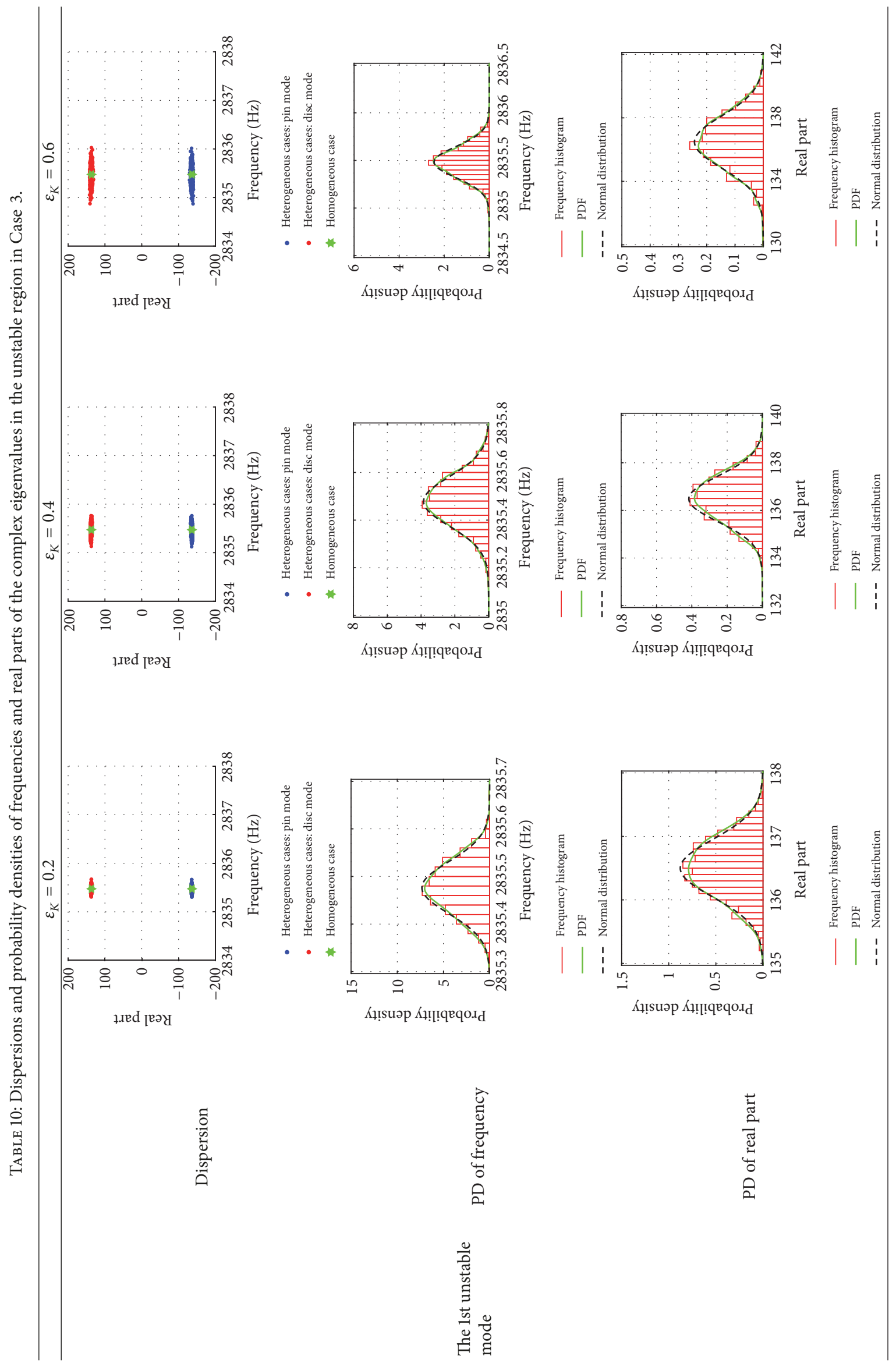




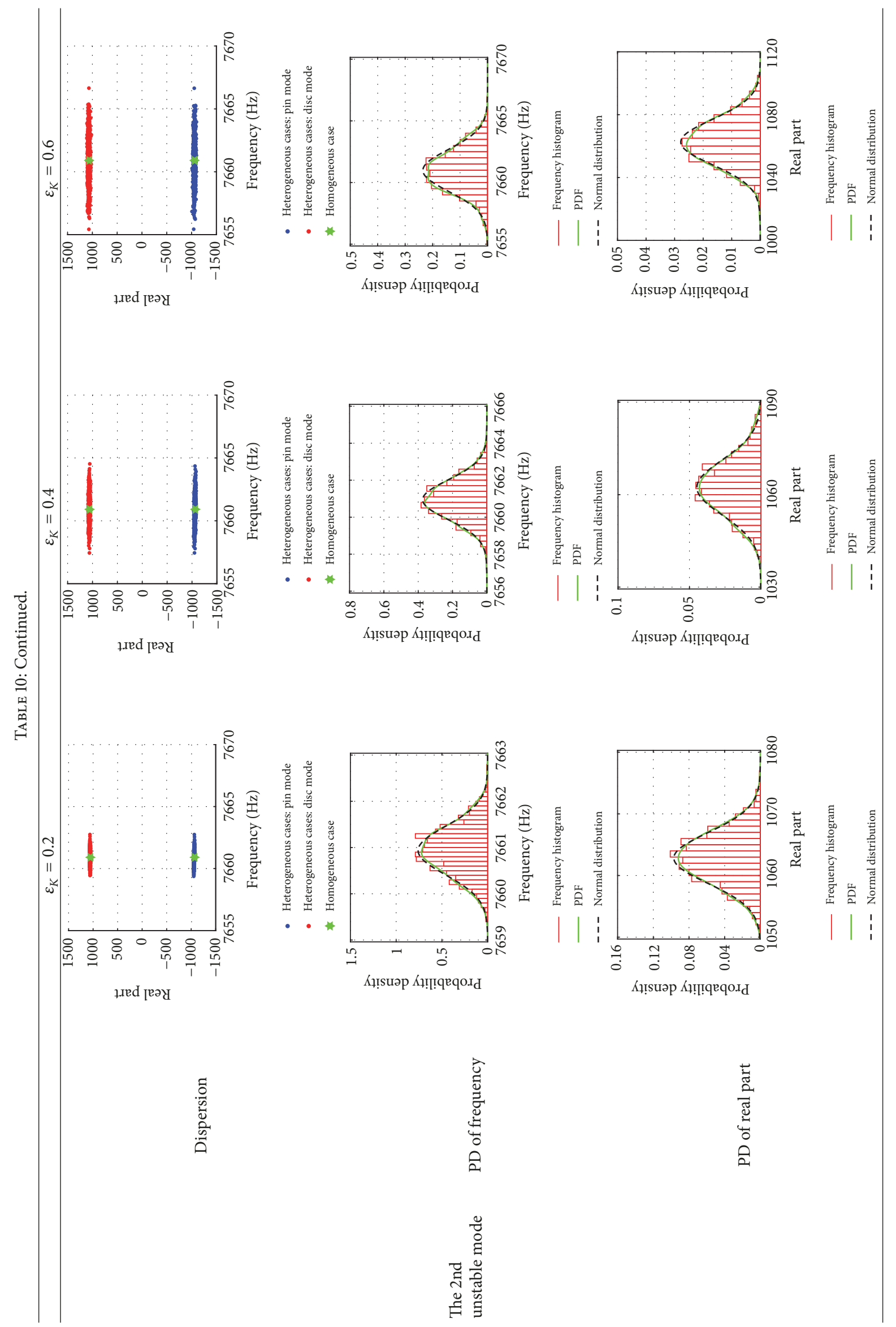




$$
\begin{aligned}
& \mathbf{B}_{N n}=-\mathbf{R}_{C}^{T} \mathbf{R}_{A 1 n}, \\
& \mathbf{A}_{t n}=\mathbf{R}_{C}^{T} \mathbf{R}_{C}, \\
& \mathbf{F}_{n}=F_{L n}\left(-\mathbf{R}_{A 2 n}^{T}+\mu_{n} \mathbf{R}_{C}^{T}\right) \\
& \left(k=0,1,2, \ldots ; m=0, \pm 1, \pm 2, \ldots, n=1,2, \ldots, n_{1}\right) .
\end{aligned}
$$

Then, making use of (A.21), (A.17), (A.18), and (A.19) can be expressed in matrix form as

$$
\begin{gathered}
\ddot{\mathbf{y}}+j 2 \Omega \mathbf{C} \dot{\mathbf{y}}-\Omega^{2} \mathbf{D} \mathbf{y}+\mathbf{C}_{s} \dot{\mathbf{y}}+j \Omega \mathbf{C C}_{s} \mathbf{y}+\left[\omega^{2}\right] \mathbf{y} \\
+\sum_{n=1}^{n_{1}} K_{N n} \mathbf{A}_{N n} \mathbf{y}+\sum_{n=1}^{n_{1}} \mu_{n} K_{N n} \mathbf{B}_{N n} \mathbf{y} \\
+\sum_{n=1}^{n_{1}} \xi K_{N n} \mathbf{A}_{t n} \mathbf{y}=\sum_{n=1}^{n_{1}} \mathbf{F}_{n} .
\end{gathered}
$$

\section{B. Parameters Used for the Pin-on-Disc System}

Parameters of pin-on-disc system are shown in Tables 3 and 4.

Other parameters are $r_{0}=0.095 \mathrm{~m}, \gamma=5^{\circ}, \Omega=20 \mathrm{rad} / \mathrm{s}$, $D^{*}=c_{t}=c_{a}=0$, and $\xi=0.2$.

\section{Conflicts of Interest}

The authors declare that there are no conflicts of interest regarding the publication of this paper.

\section{Acknowledgments}

This study was supported by the National Natural Science Foundation of China (no. 51575395).

\section{References}

[1] A. Akay, "Acoustics of friction," The Journal of the Acoustical Society of America, vol. 111, no. 4, pp. 1525-1548, 2002.

[2] N. M. Kinkaid, O. M. O'Reilly, and P. Papadopoulos, "Automotive disc brake squeal," Journal of Sound and Vibration, vol. 267, no. 1, pp. 105-166, 2003.

[3] Z. Zhou, Y. Lei, and S. Zhang, Frontier of Tribology, Science Press, Beijing, China, 2006.

[4] Engineering and Materials Science Division of NSFC, Strategy report of mechanical engineering discipline development (20112020), Science Press, Beijing, China, 2010.

[5] M. Rusli and M. Okuma, "Effect of surface topography on mode-coupling model of dry contact sliding systems," Journal of Sound and Vibration, vol. 308, no. 3-5, pp. 721-734, 2007.

[6] M. Huang, "Statistical Analysis of Impacts of Surface Topography on Brake Squeal in Disc-Pad System," SAE International Journal of Passenger Cars-Mechanical Systems, vol. 7, no. 3, 2014.

[7] F. Massi, Y. Berthier, and L. Baillet, "Contact surface topography and system dynamics of brake squeal," Wear, vol. 265, no. 11-12, pp. 1784-1792, 2008.
[8] H. A. Sherif, "Investigation on effect of surface topography of $\mathrm{pad} /$ disc assembly on squeal generation," Wear, vol. 257, no. 7-8, pp. 687-695, 2004.

[9] J. Kang, C. M. Krousgrill, and F. Sadeghi, "Analytical formulation of mode-coupling instability in disc-pad coupled system," International Journal of Mechanical Sciences, vol. 51, no. 1, pp. 52-63, 2009.

[10] U. von Wagner, D. Hochlenert, and P. Hagedorn, "Minimal models for disk brake squeal," Journal of Sound and Vibration, vol. 302, no. 3, pp. 527-539, 2007.

[11] M. T. Júnior, S. N. Y. Gerges, and R. Jordan, "Analysis of brake squeal noise using the finite element method: a parametric study," Applied Acoustics, vol. 69, no. 2, pp. 147-162, 2008.

[12] J. Kang, "Squeal analysis of gyroscopic disc brake system based on finite element method," International Journal of Mechanical Sciences, vol. 51, no. 4, pp. 284-294, 2009.

[13] N. Hoffmann, M. Fischer, R. Allgaier, and L. Gaul, "A minimal model for studying properties of the mode-coupling type instability in friction induced oscillations," Mechanics Research Communications, vol. 29, no. 4, pp. 197-205, 2002.

[14] H. Ouyang, J. E. Mottershead, M. P. Cartmell, and M. I. Friswell, "Friction-induced parametric resonances in discs: Effect of a negative friction-velocity relationship," Journal of Sound and Vibration, vol. 209, no. 2, pp. 251-263, 1998.

[15] H. Hetzler and K. Willner, "On the influence of contact tribology on brake squeal," Tribology International, vol. 46, pp. 237-246, 2012.

[16] A. Renault, F. Massa, B. Lallemand, and T. Tison, "Experimental investigations for uncertainty quantification in brake squeal analysis," Journal of Sound and Vibration, vol. 367, pp. 37-55, 2016.

[17] E. Sarrouy, O. Dessombz, and J.-J. Sinou, "Piecewise polynomial chaos expansion with an application to brake squeal of a linear brake system," Journal of Sound and Vibration, vol. 332, no. 3, pp. 577-594, 2013.

[18] H. Lü and D. Yu, "Brake squeal reduction of vehicle disc brake system with interval parameters by uncertain optimization," Journal of Sound and Vibration, vol. 333, no. 26, pp. 7313-7325, 2014.

[19] H. Lü and D. Yu, "Stability analysis and improvement of uncertain disk brake systems with random and interval parameters for squeal reduction," Journal of Vibration and Acoustics, vol. 137, no. 5, Article ID 051003, 11 pages, 2015.

[20] A. Culla and F. Massi, "Uncertainty model for contact instability prediction," The Journal of the Acoustical Society of America, vol. 126, no. 3, pp. 1111-1119, 2009.

[21] A. Nobari, H. Ouyang, and P. Bannister, "Uncertainty quantification of squeal instability via surrogate modelling," Mechanical Systems and Signal Processing, vol. 60-61, pp. 887-908, 2015.

[22] E. Sarrouy, O. Dessombz, and J.-J. Sinou, "Stochastic study of a non-linear self-excited system with friction," European Journal of Mechanics - A/Solids, vol. 40, pp. 1-10, 2013.

[23] J. Kang, C. M. Krousgrill, and F. Sadeghi, "Dynamic instability of a thin circular plate with friction interface and its application to disc brake squeal," Journal of Sound and Vibration, vol. 316, no. 1-5, pp. 164-179, 2008. 


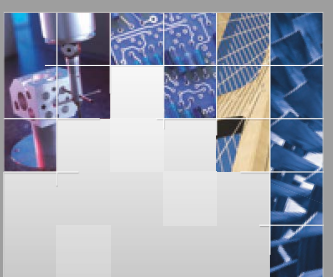

\section{Enfincering}
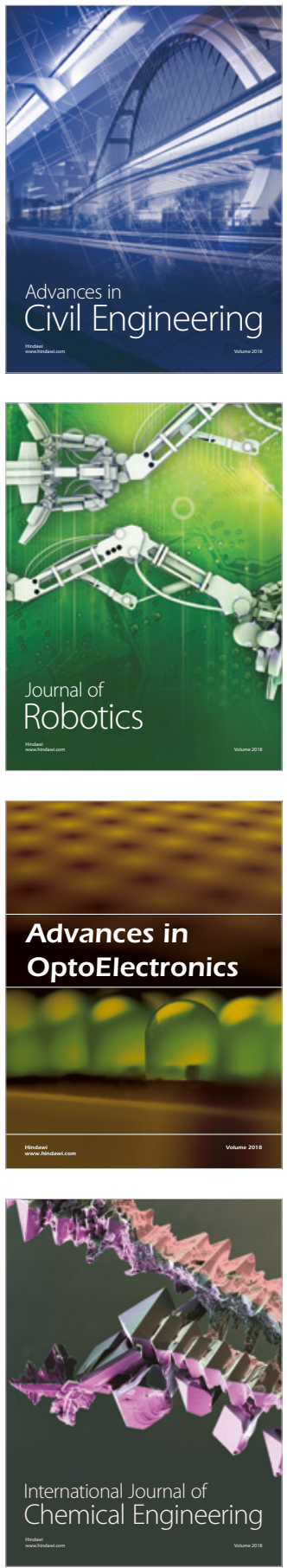

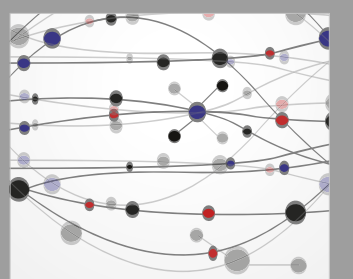

\section{Rotating \\ Machinery}

The Scientific World Journal

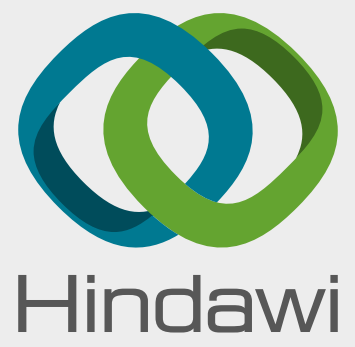

Submit your manuscripts at

www.hindawi.com
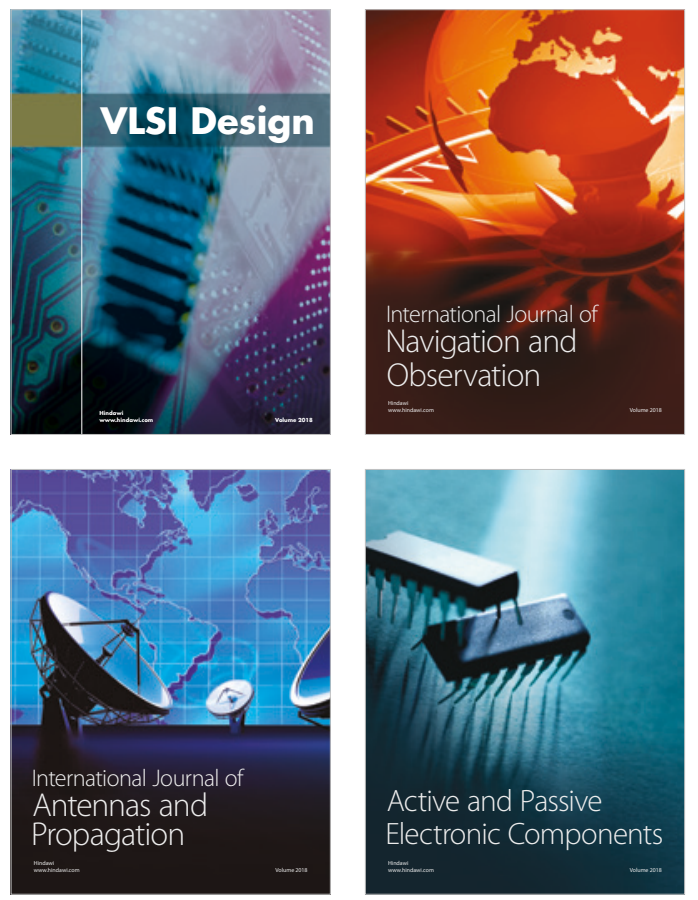
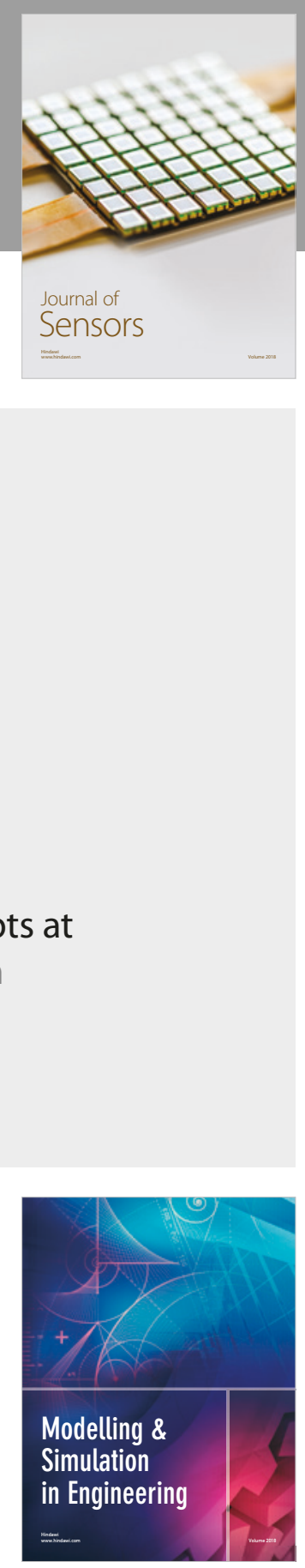

\section{Advances \\ Multimedia}
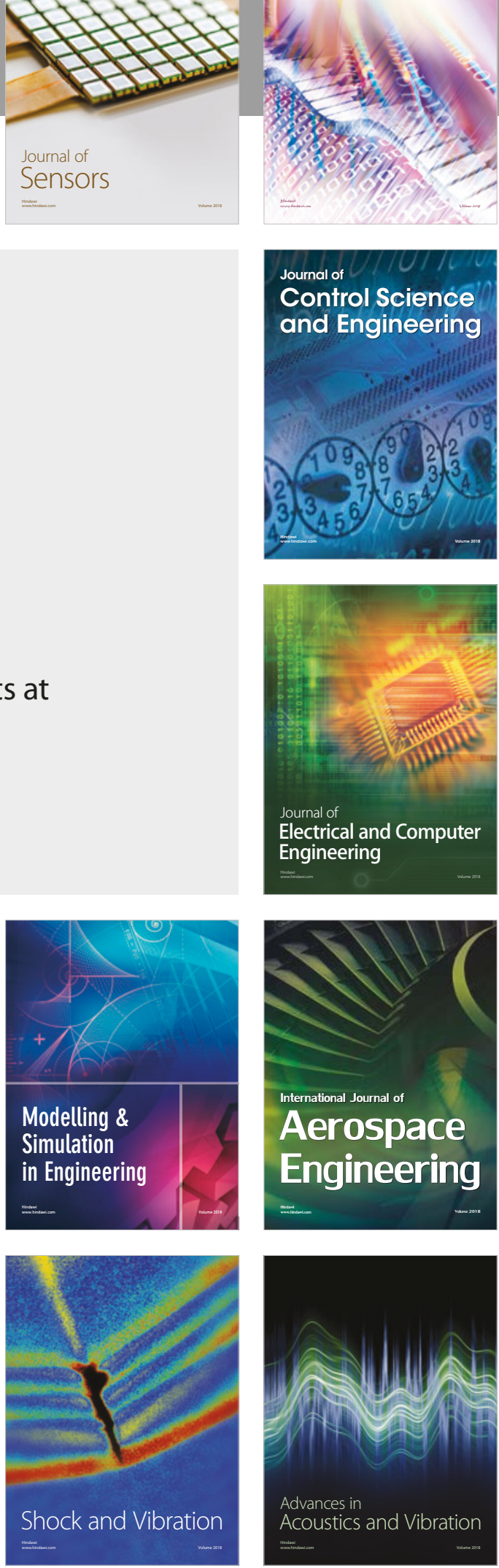